\title{
Comparative neuronal morphology of the cerebellar cortex in afrotherians, carnivores, cetartiodactyls, and primates
}

\author{
Bob Jacobs ${ }^{1 *}$, Nicholas L. Johnson ${ }^{1}$, Devin Wahl' ${ }^{1}$ Matthew Schall ${ }^{1}$, Busisiwe C. Maseko ${ }^{2}$, \\ Albert Lewandowski ${ }^{3}$, Mary A. Raghanti ${ }^{4}$, Bridget Wicinski ${ }^{5}$, Camilla Butti ${ }^{5}$, William D. Hopkins ${ }^{6}$, \\ Mads F. Bertelsen", Timothy Walsh ${ }^{8}$, John R. Roberts ${ }^{8}$, Roger L. Reep ${ }^{9}$, Patrick R. Hof ${ }^{5}$, \\ Chet C. Sherwood ${ }^{10}$ and Paul R. Manger ${ }^{2}$
}

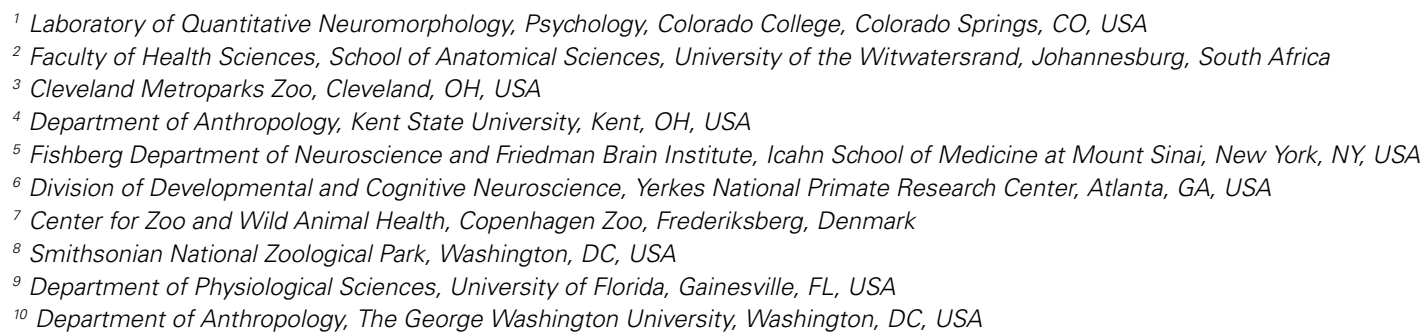

\section{Edited by:}

Suzana Herculano-Houzel,

Universidade Federal do Rio de Janeiro, Brazil

\section{Reviewed by:}

James M. Bower, University of

Texas Health Science Center San Antonio, USA

Suzana Herculano-Houzel,

Universidade Federal do Rio de

Janeiro, Brazil

Andrew Iwaniuk, University of

Lethbridge, Canada

*Correspondence:

Bob Jacobs, Laboratory of Quantitative Neuromorphology,

Psychology, Colorado College, 14 E. Cache La Poudre, Colorado Springs, CO 80903, USA

e-mail: bjacobs@

coloradocollege.edu
Although the basic morphological characteristics of neurons in the cerebellar cortex have been documented in several species, virtually nothing is known about the quantitative morphological characteristics of these neurons across different taxa. To that end, the present study investigated cerebellar neuronal morphology among eight different, large-brained mammalian species comprising a broad phylogenetic range: afrotherians (African elephant, Florida manatee), carnivores (Siberian tiger, clouded leopard), cetartiodactyls (humpback whale, giraffe) and primates (human, common chimpanzee). Specifically, several neuron types (e.g., stellate, basket, Lugaro, Golgi, and granule neurons; $N=317$ ) of the cerebellar cortex were stained with a modified rapid Golgi technique and quantified on a computer-assisted microscopy system. There was a 64-fold variation in brain mass across species in our sample (from clouded leopard to the elephant) and a 103-fold variation in cerebellar volume. Most dendritic measures tended to increase with cerebellar volume. The cerebellar cortex in these species exhibited the trilaminate pattern common to all mammals. Morphologically, neuron types in the cerebellar cortex were generally consistent with those described in primates (Fox et al., 1967) and rodents (Palay and Chan-Palay, 1974), although there was substantial quantitative variation across species. In particular, Lugaro neurons in the elephant appeared to be disproportionately larger than those in other species. To explore potential quantitative differences in dendritic measures across species, MARSplines analyses were used to evaluate whether species could be differentiated from each other based on dendritic characteristics alone. Results of these analyses indicated that there were significant differences among all species in dendritic measures.

Keywords: dendrite, morphometry, Golgi method, brain evolution, cerebellum

\section{INTRODUCTION}

In terms of gross anatomy, the cerebellum appears to have a common plan in all mammals (Bolk, 1906; Breathnach, 1955; Larsell, 1970; Sultan and Braitenberg, 1993), although absolute and relative size can vary considerably (Marino et al., 2000; Maseko et al., 2012b). Histologically, cerebellar cortex exhibits a generally trilaminate architecture, which is similar in birds and mammals (Ramón y Cajal, 1909, 1911; Iwaniuk et al., 2006; Sultan and Glickstein, 2007). Whereas limited aspects of cerebellar neuron morphology have been described in some vertebrate species (e.g., mormyrid electric fish: Han et al., 2006; teleost fish: Murakami and Morita, 1987; alligator: Nicholson and Llinas, 1971; cat:
Melik-Musyan and Fanardzhyan, 2004; duck: O'Leary et al., 1968; dolphin: Adanina, 1965; rhesus monkey: Fox et al., 1967; Rakic, 1972; human: Braak and Braak, 1983), the most detailed research has focused on rodents. In particular, Palay and Chan-Palay (1974) provided a comprehensive examination of the cerebellar cortex of the rat, documenting organizational features, neuronal morphology, and ultrastructure at the electron microscopic level. Recently, we expanded the scope of such investigations with an examination of neuronal morphology in the cerebellar cortex of the African elephant (Maseko et al., 2012a). The current, rapid Golgi study is part of a larger project to document neuronal morphology of both the cerebral neocortex (Jacobs et al., 
2011) and the cerebellar cortex in large brained mammals not previously examined. Such comparative investigations may help discern which aspects of neuronal morphology are general to all vertebrates, and which are specific to particular species (Meek et al., 2008). To this end, we examine cortical neuronal morphology in the cerebella of eight different mammalian species comprising four diverse taxa: afrotherians (African elephant, Florida manatee), carnivores (Siberian tiger, clouded leopard), cetartiodactyls (humpback whale, giraffe), and primates (human, common chimpanzee).

Although there are many representative freehand and camera lucida drawings of cerebellar cortex neurons (Ramón y Cajal, 1909, 1911; Chan-Palay and Palay, 1970, 1972; Palay and ChanPalay, 1974; Braak and Braak, 1983; Bishop, 1993; Lainé and Axelrad, 1996), very few cerebellar neurons have been digitally reconstructed relative to those in the neocortex and hippocampus (Halavi et al., 2012). In fact, it is revealing that, of the 10,004 digital reconstructions currently in the online repository at Neuromorpho.org, only 24 are cerebellar neurons (as opposed to 5405 cerebral cortex neurons). In terms of digital reconstructions, the Purkinje neuron has been traced much more than other cerebellar neurons, perhaps because of its central role as the sole output neuron for the cerebellar cortex in tetrapods (Marr, 1969; Dean et al., 2010). The most complete Purkinje cell tracings are typically the result of injection techniques (e.g., Lucifer yellow: Sawada et al., 2010; biocytin: Roth and Häuser, 2001), and immunohistochemistry (Wu et al., 2010) with confocal laser microscropy, although the number of reconstructions usually remains small $(<30)$. An even more limited number of Purkinje neuron reconstructions have been obtained using horseradish peroxidase and Golgi-Cox impregnations with light microscopy (Calvet and Calvet, 1984; Rapp et al., 1994; Milatovic et al., 2010). There appear to be no digital reconstructions of Purkinje neurons based on rapid Golgi stains. Finally, apart from a small number of traced molecular layer interneurons $(N=26$; Sultan and Bower, 1998), there are few complete digital reconstructions of other neuronal types in cerebellar cortex.

In terms of comparative neuromorphology, research has generally focused on qualitative descriptions of Purkinje neurons. For example, there are well-documented morphological differences between tetrapods and teleosts such as the mormyrids, which have Purkinje neuron dendrites with a distinct palisade pattern (Meek and Nieuwenhuys, 1991). Quantitatively, however, there is very little comparative morphological information on cerebellar cortical neurons. To this end, the present study documents the morphological attributes of several types of cerebellar neurons. Following descriptions in rodents (Palay and Chan-Palay, 1974) and other mammals (rhesus monkey: Fox et al., 1967; cat: Larsell and Jansen, 1972; human: Braak and Braak, 1983), the superficial molecular layer contains the two-dimensional dendritic arrays of Purkinje neurons. These Purkinje neurons are described only qualitatively in the present study because rapid Golgi impregnations under light microscopy make complete and accurate tracings of their dense, distal dendritic segments extremely problematic, if not impossible. Also in the molecular layer are inhibitory interneurons, classically divided into (1) the relatively small stellate neurons in the outer two thirds of the layer, which are characterized by contorted, frequently dividing dendritic trees that radiate in multiple directions and by axons that are generally oriented horizontally; and (2) the somewhat deeper basket neurons, characterized by extensive, sea-fan shaped dendritic arbors and horizontally oriented axons that terminate in multiple pericellular baskets around the somata of Purkinje neurons. Although we follow the classical terminology for these interneurons in the present paper, it should be noted that both developmental research (Rakic, 1972) and empirical investigations (Sultan and Bower, 1998; Leto et al., 2006; Schilling et al., 2009) support early speculation (Ramón y Cajal, 1909, 1911) that these molecular inhibitory interneurons may actually be a uniform cell type whose ultimate morphology is determined by local cues at particular depths of the molecular layer.

Under the molecular layer, the Purkinje cell layer contains the large somata of Purkinje neurons, arranged in a single row, providing a clear demarcation between the other two layers. The deep granule cell layer contains the somata of two relatively large interneurons: (1) located immediately beneath the Purkinje cell layer, the Lugaro neurons (Golgi, 1874; Lugaro, 1894) are characterized by triangular or elongated fusiform shaped somata from which relatively long, thick, unbranched dendrites originate, typically extending in an arc under the Purkinje cell layer; and (2) somewhat deeper in the granule cell layer, the Golgi neurons (Golgi, 1874) are characterized by round somata with multiple dendrites radiating in all directions. Finally, throughout the granule cell layer are the very densely packed granule neurons, characterized by small, round somata extending several short, relatively unbranched dendrites characterized by gnarled, claw-like terminations.

The goals of the present comparative study were three-fold: (1) provide a qualitative description of neuronal morphology in the cerebellar cortex across the eight species examined; (2) provide quantitative data on the dendritic characteristics of these neurons; and (3) examine potential species differences in the dendritic measures of the traced neurons.

\section{MATERIALS AND METHODS SPECIMENS}

Tissue was obtained from eight species in the following phylogenetic groups: afrotherians (African elephant, Florida manatee), carnivores (Siberian tiger, clouded leopard), cetartiodactyls (humpback whale, giraffe), and primates (human, common chimpanzee). For captive animals (Siberian tiger, clouded leopard, chimpanzee), observations prior to death revealed no obvious behavioral abnormalities or deficits. Similar observations were not possible for animals in their natural habitat (African elephant, giraffe, humpback whale, Florida manatee, human). In post-mortem examinations, the brains of all animals exhibited no obvious abnormalities in terms of gross neuroanatomy. For five species (African elephant, Siberian tiger, clouded leopard, humpback whale, giraffe), cerebellar volume for at least one of the animals was obtained through magnetic resonance imaging (Maseko et al., 2012b). For the other species (Florida manatee, human, chimpanzee), direct measurement was not obtained because we did not have the opportunity for MRI scanning, nor was destructive dissection of the cerebellum an option. Instead, 
we had to rely on species mean values from the published literature. The present study was approved by the Colorado College Institutional Review Board (\#011311-1) and the University of the Witwatersrand Animal Ethics Committee (2008/36/1).

\section{African elephant (Loxodonta africana)}

Cerebellar tissue from two 20 to 30-year-old, solitary male African elephants scheduled for population management culling was obtained after they were euthanized as described in Manger et al. (2009). In situ perfusion-fixation of the brains was conducted by removal of the head, flushing of the head with cold saline, and intra-carotid perfusion with $4 \%$ paraformaldehyde in $0.1 \mathrm{M}$ phosphate buffer (autolysis time, AT, averaged $=135 \mathrm{~min}$ ). The brains were then removed from the skull, placed in the same cold fixative and stored in $4 \%$ paraformaldehyde in $0.1 \mathrm{M}$ phosphate buffer for $72 \mathrm{~h}$. One brain had a mass of $5145 \mathrm{~g}$ and a cerebellar volume of $946 \mathrm{ml}$; the other brain had a mass of $4835 \mathrm{~g}$ and a cerebellar volume of $902 \mathrm{ml}$ (Maseko et al., 2012b). Small tissue blocks containing the cerebellar regions of interest were stored in $0.1 \%$ sodium azide in $0.1 \mathrm{M}$ phosphate buffer saline at $4^{\circ} \mathrm{C}$ for 8 months before Golgi staining.

\section{Florida manatee (Trichechus manatus latirostris)}

Following a watercraft collision in Florida, a sub-adult female manatee was euthanized. The head was perfused (by Roger L. Reep) via bilateral cannulation of the internal carotids, with 201 phosphate buffer followed by 101 of $4 \%$ paraformaldehyde. The brain (brain mass $=316$ g; estimated cerebellar volume $=44 \mathrm{ml}$; Reep and O'Shea, 1990) was removed (AT $=6 \mathrm{~h})$ and stored in a cold $2 \%$ paraformadehyde solution for $\sim 2$ days. One cerebellar tissue block was removed and stored in cold $\left(2^{\circ} \mathrm{C}\right)$ phosphate buffer solution for 3 additional days before Golgi staining.

\section{Siberian tiger (Panthera tigris altaica)}

One 12-year-old female from the Copenhagen Zoo in Denmark was euthanized. In situ perfusion-fixation (by Mads F. Bertelsen) of the brain (AT $<30 \mathrm{~min}$ ) followed the same protocol as in the elephant (brain mass $=258 \mathrm{~g}$; cerebellar volume $=37 \mathrm{ml}$ ). Cerebellar tissue blocks were stored in $0.01 \%$ sodium azide in $0.1 \mathrm{M}$ phosphate buffer saline at $4^{\circ} \mathrm{C}$ for 6 months before Golgi staining.

\section{Clouded leopard (Neofelis nebulosa)}

Two adult female clouded leopards were euthanized for medical reasons: a 20-year old from the Smithsonian National Zoological Park in Washington, DC., and a 28-year old from the Cleveland Metroparks Zoo (AT $<30 \mathrm{~min}$ for both animals). The brains were immersion fixed in 10\% formalin for 10 (20-year old) and 34 days (28-year old). Brain mass was $82 \mathrm{~g}$ for the 20 -year old and $73 \mathrm{~g}$ for the 28-year old; cerebellar volume was an average of $8.6 \mathrm{ml}$ for both animals. Subsequently, the brains were stored in $0.1 \%$ sodium azide in $0.1 \mathrm{M}$ phosphate buffer saline at $4^{\circ} \mathrm{C}$ prior to Golgi staining ( 5 months for the 20 -year old; 3 years for the 28-year old).

\section{Humpback whale (Megaptera novaeangliae)}

A 2 year-old male humpback whale, $9.45 \mathrm{~m}$ in length, was stranded in East Hampton, Long Island, New York in April, 2010.
A necropsy was performed (by Patrick R. Hof, Bridget Wicinski, and Camilla Butti) immediately after death. The brain (brain mass $=3606 \mathrm{~g}$; cerebellar volume $=695 \mathrm{ml}$ ) was removed (AT $=8 \mathrm{~h}$ ) and immersion-fixed in $4 \%$ paraformaldehyde for 2 years prior to Golgi staining.

\section{Giraffe (Giraffa camelopardalis)}

The brains of three solitary, free ranging, sub-adult ( $\sim 2-3$ years of age) male giraffes were obtained and processed in the same manner as the elephant (Dell et al., 2012). Brain masses-cerebellar volumes for these three animals were $610 \mathrm{~g}-83 \mathrm{ml}, 527 \mathrm{~g}-69 \mathrm{ml}$, and $480 \mathrm{~g}-67 \mathrm{ml}$. Cerebellar blocks were stored in $0.1 \%$ sodium azide in $0.1 \mathrm{M}$ phosphate buffer for 4 months prior to Golgi staining.

\section{Human (Homo sapiens)}

Human tissue was provided by Dr. R. Bux of the El Paso County coroner's office in Colorado Springs. Tissue blocks were removed from the cerebellum of a neurologically normal, 54-year-old male who had died of acute myocardial infarction (brain mass = 1435 g; estimated cerebellar volume $=139$ ml; Smaers et al., 2011; Maseko et al., 2012b). Tissue was immersion fixed in $10 \%$ formalin and stored at $2^{\circ} \mathrm{C}$ for $\sim 1$ week before Golgi staining $(\mathrm{AT}=5 \mathrm{~h})$.

\section{Common chimpanzee (Pan troglodytes)}

Two adult chimpanzees were obtained from the Yerkes National Primate Center: a 23-year-old female who died under anesthesia, and a 39-year-old male euthanized due to congestive heart failure. Brains were immersion fixed in $10 \%$ formalin (13 days for the 23-year old; 4 months for the 39 year old; AT $<1 \mathrm{~h}$ ). Subsequently, brains were stored in $0.1 \%$ sodium azide in $0.1 \mathrm{M}$ phosphate buffer saline at $4^{\circ} \mathrm{C}$ prior to Golgi staining ( 4 years for the 23-year old; 2 years for the 39-year old). Brain mass was $408 \mathrm{~g}$ for the 23-year old and $392 \mathrm{~g}$ for the 39-year old; cerebellar volume was estimated to be an average of $43 \mathrm{ml}$ for both animals (Smaers et al., 2011; Maseko et al., 2012b).

\section{TISSUE SELECTION}

In five of the species (Florida manatee, Siberian tiger, humpback whale, human, and chimpanzee), tissue blocks (3-5 mm thick) were removed from the dorsal posterior aspect of the posterior lobe and from the dorsal anterior aspect of the anterior lobe of the left cerebellar hemisphere (Figure 1). In the remaining three species (African elephant, giraffe, and clouded leopard), the same regions were sampled from the right cerebellar hemisphere. Tissue was coded to prevent experimenter bias, stained via a modified rapid Golgi technique (Scheibel and Scheibel, 1978), and sectioned serially perpendicular to the long axis of the folia at $120 \mu \mathrm{m}$ with a vibratome (Leica VT1000S, Leica Microsystems, Inc.). Because of the small number of neurons traced in each species, neurons from anterior and posterior cerebellar lobes were combined for all subsequent analyses.

\section{NEURON SELECTION AND QUANTIFICATION}

Neurons were selected for tracing based on established criteria (Roitman et al., 2002; Anderson et al., 2009; Jacobs et al., 2011; Lu et al., 2013), which required an isolated, darkly stained soma near 


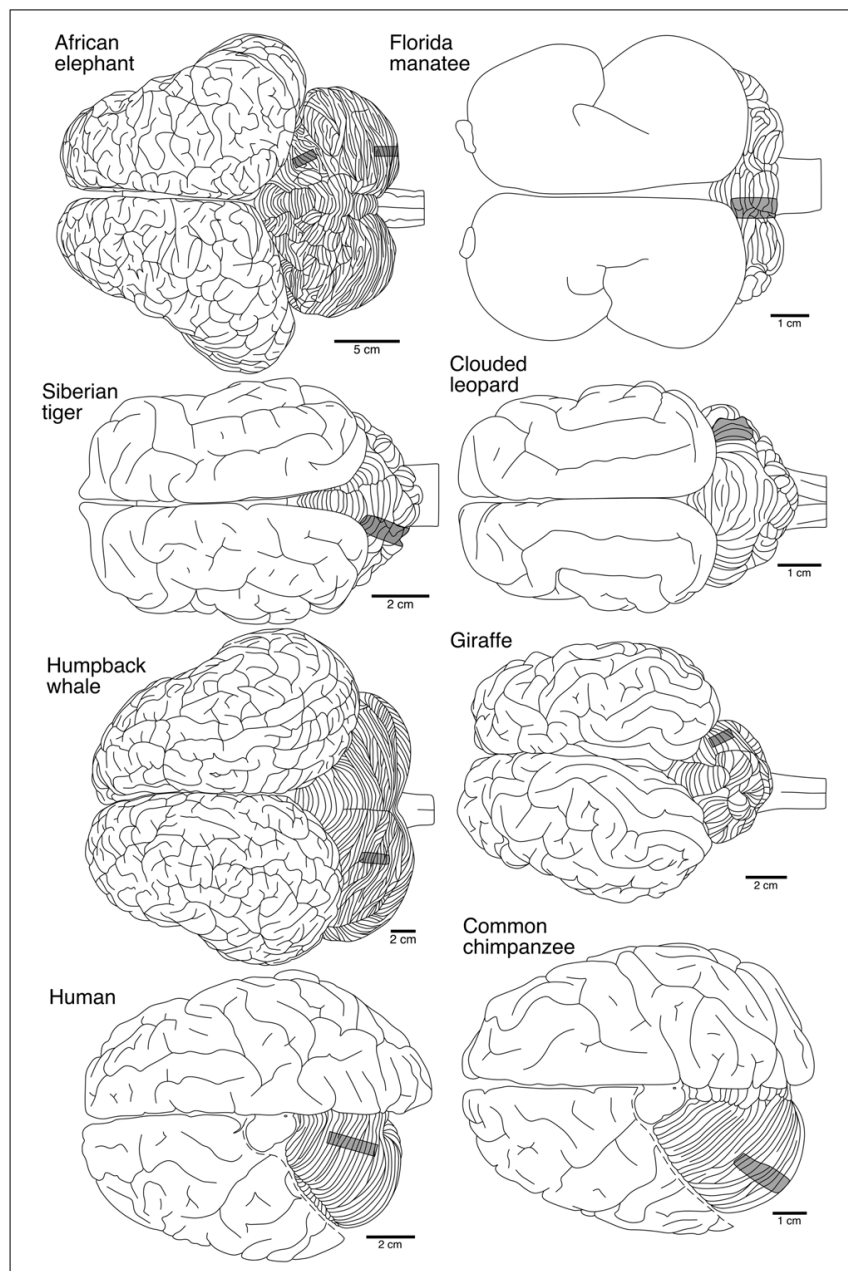

FIGURE 1 | Dorsal views of the brains from the eight species in the current study illustrating the relative location from which tissue blocks were selected from the cerebellum for staining. Represented from top to bottom are: afrotherians (African elephant, Florida manatee), carnivores (Siberian tiger, clouded leopard), cetartiodactyls (humpback whale, giraffe), and primates (human, common chimpanzee). For the two primates, the dorsal portion of the cerebrum has been removed to reveal the cerebellum. Note scale bar is different for each species. the center of the $120 \mu \mathrm{m}$ section, with as fully impregnated, unobscured, and complete dendritic arbors as possible (i.e., no beading or interruptions). In the tracing process, dendritic branches were not followed into adjacent sections. Although serial section reconstructions of dendrites are possible with some histological techniques (e.g., intracellular injections), accurate reconstructions are problematic in Golgi stained material, where multiple neural elements overlap in the same section. As such, only portions captured in the $120 \mu \mathrm{m}$-thick section could be compared in the present study, resulting in an overall underestimation of dendritic values insofar as neurons with longer dendrites are disproportionately cut in the sectioning process. Prior to quantification, Golgi-stained sections were examined to determine neuronal types. The neurons of interest included the molecular layer interneurons (e.g., stellate, basket), as well as Lugaro,
Golgi, and granule cells. As noted above, we kept the classical distinction between stellate and basket neurons for the molecular layer interneurons, although it has been argued that they actually constitute the same neuronal population (Sultan and Bower, 1998). Additionally, no distinction was made between superficial and deeper stellate neurons, or between large and small Golgi neurons (Palay and Chan-Palay, 1974). Purkinje neurons were photomicrographed but not traced due to the complexity of their dendritic plexus. Candelabrum (Lainé and Axelrad, 1994), unipolar brush (Altman and Bayer, 1977), and synarmotic (Landau, 1933; Flace et al., 2004) neurons were not observed in the current preparations. Certain morphological characteristics of neurons traced in the elephant have previously been reported (Maseko et al., 2012a), but are included here with a more in-depth analysis. Golgi impregnation was inconsistent across species, both in terms of overall numbers of neurons and the types of neurons that stained. Consequently, neurons traced in different individuals within a species (i.e., elephant, clouded leopard, giraffe, chimpanzee) were combined without consideration of individual differences.

Quantification was performed under a Planachromatic 60x oil objective (N.A. 1.4), except for elephant neurons, which were quantified under a Planachromat 40x dry objective (N.A. $0.70)$. Based on prior research, this difference in microscope objectives was not expected to significantly affect dendritic measures (Anderson et al., 2010). Neurons were traced along X-, $\mathrm{y}-, \mathrm{z}$-coordinates using a Neurolucida system (MBF Bioscience, Williston, VT) interfaced with an Olympus BH-2 microscope equipped with a Ludl XY motorized stage (Ludl Electronics, Hawthorne, NY) and a Heidenhain z-axis encoder (Schaumburg, IL). A MicroFire Digital CCD 2-megapixel camera (Optronics, Goleta, CA) mounted on a trinocular head (model 1-L0229, Olympus, Center Valley, PA) displayed images on a $1920 \times 1200$ resolution Dell E248WFP 24-inch LCD monitor. Somata were traced first at their widest point in the 2-dimensional plane to provide an estimate of the cross-sectional soma area, a measure that appears highly correlated with soma volume (Ulfhake, 1984). Subsequently, dendrites were traced somatofugally in their entirety, recording dendritic diameter. Dendritic arbors with unclear, sectioned, broken, ambiguous, or obscured terminations were identified as incomplete endings. Of the 13,698 dendritic segments quantified, $45 \%$ were intermediate segments. With regard to terminal segments, $58 \%$ were complete, and $42 \%$ were incomplete, which is a higher completion ratio than obtained in neocortex with the same methodology (Jacobs et al., 1997, 2001). Neurons with sectioned segments were not differentially analyzed because elimination of neurons with incomplete segments would have biased the sample toward smaller neurons (Schadé and Caveness, 1968; Uylings et al., 1986).

Neurons were traced by three investigators (Busisiwe C. Maseko, Nicholas L. Johnson, Devin Wahl). Intrarater reliability was determined by having each rater trace the same soma and dendritic segment 10 times. The average coefficient of variation for soma size (2.5\%) and total dendritic length (TDL, 2.8\%) indicated little variation in the tracings. Intrarater reliability was further tested with a split plot design $(\alpha=0.05)$, which indicated no significant difference between the first five tracings and the last 
five tracings. Interrater reliability was determined through comparison of 10 dendritic system tracings with the same tracings completed by the primary investigator (Bob Jacobs). Interclass correlations across soma size and TDL averaged 0.99 and 0.99, respectively. An analysis of variance (ANOVA; $\alpha=0.05$ ) failed to indicate significant differences among the tracers for the three measures. Additionally, the primary investigator reexamined all completed tracings under the microscope to ensure accuracy.

\section{CELL DESCRIPTIONS AND DEPENDENT DENDRITIC MEASURES}

Neurons were classified according to somatodendritic morphological characteristics, closely following well-established descriptive criteria (Ramón y Cajal, 1909, 1911; Rakic, 1972; Palay and Chan-Palay, 1974; Braak and Braak, 1983; Melik-Musyan and Fanardzhyan, 2004). Quantitatively, soma size (i.e., surface area, $\left.\mu \mathrm{m}^{2}\right)$ and depth from the pial surface $(\mu \mathrm{m})$ were measured. Dendritic branches extending from the soma were characterized centrifugally (Bok, 1959; Uylings et al., 1986), and quantified with four previously established measures (Jacobs et al., 2011): dendritic volume (Vol, $\mu \mathrm{m}^{3}$; the total volume of all dendrites); total dendritic length (TDL, $\mu \mathrm{m}$; the summed length of all dendritic segments); mean segment length (MSL, $\mu \mathrm{m}$; the average length of each dendritic segment); and dendritic segment count (DSC; the number of dendritic segments). One additional measure from Maseko et al. (2012a) was examined: dendritic tortuosity (Tor), a measure of the relative straightness-twistedness of a dendrite. The tortuosity index was calculated by dividing the total length of dendritic segments from the origin point on the soma to the end point by the length of a vector: an index of 1 equals a straight line; an index greater than 1 means the path of the dendrites is more complex than a straight line (Foster and Peterson, 1986; Wen et al., 2009). Finally, dendritic branching patterns were analyzed using a Sholl analysis (Sholl, 1953), which quantified dendritic intersections at $20-\mu \mathrm{m}$ intervals radiating somatofugally.

\section{INFERENTIAL STATISTICAL ANALYSES OF INTERSPECIES DIFFERENCES}

Measures (Vol, TDL, MSL, DSC, Tor) for every dendritic segment, along with soma size and depth, were aggregated for each neuron (SPSS release 20.0.0). A total of 317 neurons were traced in 12 members of 8 species, with the following breakdown: African elephant ( $n=20 ; 18$ in one animal, 2 in the other), Florida manatee $(n=25)$, Siberian tiger $(n=33)$, clouded leopard ( $n=32 ; 21$ in the 20-year old; 11 in the 28-year old), humpback whale $(n=47)$, giraffe $(n=56 ; 21,21$, and 14 in each of the three animals), chimpanzee $(n=86 ; 51$ in the 23 year old; 35 in the 39-year old), human $(n=28)$. As indicated in Table 1, neuron types were unevenly distributed among species and some neuron types did not stain in some species (i.e., granule neurons in afrotherians, and Golgi neurons in the manatee).

For inferential analyses, we initially constructed a table of dendritic measures for all neuron types to explore differences among species (Table 2). Given that cerebellar volume increases with the size of the brain (Smaers et al., 2011; Maseko et al., $2012 \mathrm{~b}$ ), the table was used to evaluate if the same is true for dendritic measures. Confidence intervals of $95 \%$ were constructed
Table 1 | Number of tracings for each neuronal type within each species.

\begin{tabular}{lrrrrrr}
\hline \multirow{2}{*}{ Species } & \multicolumn{6}{c}{ Neuron type } \\
\cline { 2 - 7 } & Stellate & Basket & Lugaro & Golgi & Granule & Total \\
\hline African elephant & 5 & 5 & 5 & 5 & 0 & 20 \\
Florida manatee & 6 & 8 & 1 & 0 & 0 & 15 \\
Siberian tiger & 5 & 8 & 3 & 7 & 10 & 33 \\
Clouded leopard & 16 & 5 & 4 & 1 & 6 & 32 \\
Humpback whale & 9 & 11 & 11 & 6 & 10 & 47 \\
Giraffe & 17 & 13 & 4 & 7 & 15 & 56 \\
Human & 7 & 6 & 5 & 5 & 5 & 28 \\
Common chimpanzee & 20 & 16 & 17 & 17 & 16 & 86 \\
\hline Total & 85 & 72 & 50 & 48 & 62 & 317 \\
\hline
\end{tabular}

for each measure. Because elephants had the largest cerebellar volume (averaging $924 \mathrm{ml}$ ) and clouded leopards the smallest (averaging $8.6 \mathrm{ml}$ ), these two species were used as the references against which the confidence intervals for the other species were compared. Unfortunately, because only one Golgi neuron stained in the clouded leopard, we could not construct confidence intervals for that species and neuron; instead, only the elephant was used as a reference for Golgi neurons. Similarly, the elephant had no stained granule neurons, so only the clouded leopard was used for comparison. In examining the table columns for each type of dendritic measure, any species-specific value outside the reference indicates a difference between the two distributions. The goal was to evaluate how much, or how little, the reference distributions for elephant and clouded leopard overlapped with the distributions of the dendritic values for the other species. By comparing the amount of overlap between the distributions, a much better idea of the comparative distributions was provided than if just $F$ tests based on means had been presented. Significant differences were highlighted in bold for comparison to the elephant and red for comparisons with the clouded leopard. Because of the usage of $95 \%$ confidence intervals, any two distributions that did not overlap were different at $p \leq 0.05$. For example, in Table 2, the TDL lower (176.5) and upper confidence intervals (2735.2) for Golgi neurons in the chimpanzee are in bold text, indicating that the Golgi TDL values in the chimpanzee $(1455.9 \pm 639.7 \mu \mathrm{m})$ are significantly $\left[F_{(1315)}=720.62, p \leq 0.05\right]$ different from those in the reference animal, namely the elephant (Golgi TDL $=5664.2 \pm$ $878.9 \mu \mathrm{m}$ ). Although Table 2 provides detailed information, it does not specify whether any of the species can be identified from just dendritic characteristics. Because accurate differentiation of species may require combinations of multiple dendritic measures, a more comprehensive analysis was necessary.

There are six potential analytic obstacles to overcome in the present, or any, quantitative neuromorphological analysis. First, the brains for each species were fixed and preserved differently, which contributed to "noise," or error variability in measurements both within the same species and between different species. Second, differential stain impregnation introduced measurement error at the dendritic level. Third, some neuron types did not stain in some animals. Fourth, the standard assumption 
Table 2 | Dendritic measures and soma size for each neuronal type across species. ${ }^{a}$

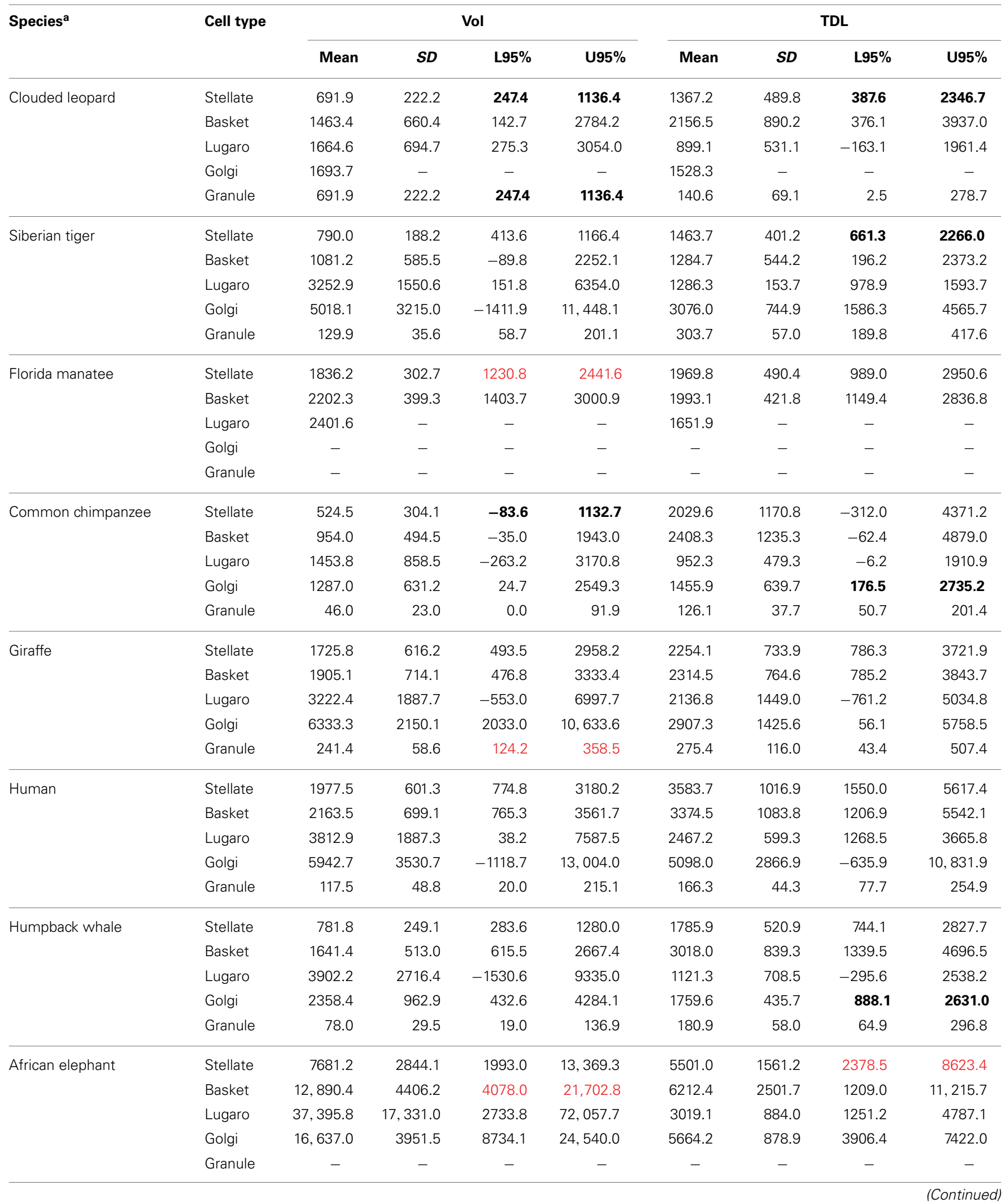


Table 2 | Continued

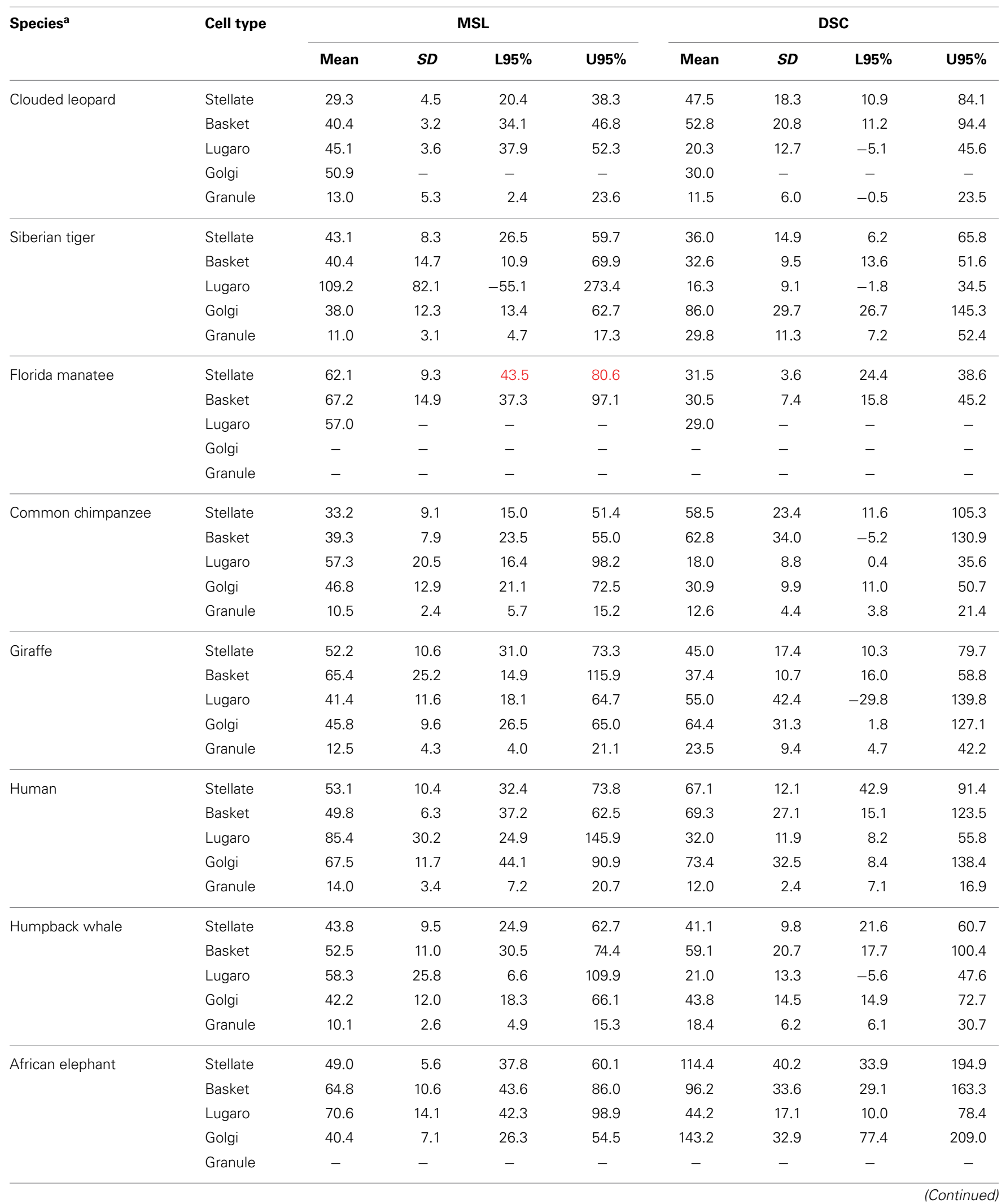


Table 2 | Continued

\begin{tabular}{|c|c|c|c|c|c|c|c|c|c|}
\hline Species $^{\mathrm{a}}$ & Cell type & \multicolumn{4}{|c|}{ Tor } & \multicolumn{4}{|c|}{ Soma size } \\
\hline \multirow{3}{*}{ Clouded leopard } & Basket & 1.11 & 0.03 & 1.05 & 1.16 & 143.8 & 11.8 & 120.2 & 167.4 \\
\hline & Lugaro & 1.11 & 0.03 & 1.05 & 1.17 & 359.3 & 112.6 & 134.1 & 584.6 \\
\hline & Golgi & 1.16 & - & - & - & 242.0 & - & - & - \\
\hline \multirow[t]{4}{*}{ Siberian tiger } & Stellate & 1.15 & 0.05 & 1.05 & 1.24 & 125.2 & 13.2 & 98.7 & 151.7 \\
\hline & Basket & 1.12 & 0.05 & 1.03 & 1.22 & 148.2 & 21.9 & 104.4 & 192.0 \\
\hline & Lugaro & 1.11 & 0.03 & 1.05 & 1.16 & 384.9 & 140.0 & 104.8 & 665.0 \\
\hline & Golgi & 1.11 & 0.02 & 1.07 & 1.14 & 480.1 & 94.1 & 291.8 & 668.3 \\
\hline \multirow{3}{*}{ Florida manatee } & Lugaro & 1.20 & - & - & - & 270.0 & - & - & - \\
\hline & Golgi & - & - & - & - & - & - & - & - \\
\hline & Granule & - & - & - & - & - & - & - & - \\
\hline \multirow[t]{5}{*}{ Common chimpanzee } & Stellate & 1.13 & 0.03 & 1.07 & 1.20 & 86.3 & 23.0 & 40.3 & 132.2 \\
\hline & Basket & 1.11 & 0.02 & 1.06 & 1.15 & 114.6 & 29.5 & 55.5 & 173.7 \\
\hline & Lugaro & 1.13 & 0.04 & 1.05 & 1.20 & 271.8 & 63.8 & 144.1 & 399.4 \\
\hline & Golgi & 1.13 & 0.02 & 1.09 & 1.17 & 285.6 & 92.5 & 100.5 & 470.7 \\
\hline & Granule & 1.26 & 0.17 & 0.92 & 1.61 & 52.9 & 5.4 & 42.1 & 63.7 \\
\hline Giraffe & Stellate & 1.16 & 0.03 & 1.10 & 1.23 & 116.5 & 25.4 & 65.8 & 167.2 \\
\hline \multirow{3}{*}{ Human } & Lugaro & 1.12 & 0.03 & 1.07 & 1.17 & 315.5 & 153.0 & 9.5 & 621.5 \\
\hline & Golgi & 1.14 & 0.02 & 1.10 & 1.18 & 433.1 & 185.1 & 63.0 & 803.3 \\
\hline & Granule & 1.51 & 0.39 & 0.72 & 2.29 & 49.7 & 6.9 & 35.9 & 63.6 \\
\hline \multirow[t]{5}{*}{ Humpback whale } & Stellate & 1.10 & 0.04 & 1.03 & 1.18 & 101.8 & 27.3 & 47.2 & 156.4 \\
\hline & Basket & 1.10 & 0.02 & 1.06 & 1.14 & 150.6 & 52.6 & 45.4 & 255.9 \\
\hline & Lugaro & 1.12 & 0.05 & 1.03 & 1.22 & 541.1 & 336.2 & -131.4 & 1213.5 \\
\hline & Golgi & 1.13 & 0.04 & 1.06 & 1.21 & 495.5 & 372.6 & -249.7 & 1240.7 \\
\hline & Granule & 1.17 & 0.08 & 1.01 & 1.33 & 63.9 & 12.0 & 39.8 & 88.0 \\
\hline \multirow[t]{5}{*}{ African elephant } & Stellate & 1.29 & 0.14 & 1.01 & 1.57 & 50.7 & 6.7 & 37.3 & 64.2 \\
\hline & Basket & 1.15 & 0.05 & 1.06 & 1.24 & 443.8 & 189.7 & 64.3 & 823.2 \\
\hline & Lugaro & 1.13 & 0.05 & 1.03 & 1.23 & 1353.7 & 394.7 & 564.4 & 2143.1 \\
\hline & Golgi & 1.19 & 0.02 & 1.14 & 1.23 & 736.5 & 131.9 & 472.6 & 1000.3 \\
\hline & Granule & - & - & - & - & - & - & - & - \\
\hline
\end{tabular}

a Dependent measures are: Volume (Vol, $\mu \mathrm{m}^{3}$ ), Total Dendritic Length (TDL, $\left.\mu \mathrm{m}\right)$, Mean Segment Length (MSL), Dendritic Segment Count (DSC), Tortuosity (Tor), and Soma Size $\left(\mu \mathrm{m}^{2}\right.$ ). Species are arranged from smallest (clouded leopard) to largest (African elephant) in terms of brain mass. L95\% $=$ lower $95 \%$ confidence interval; U95\% = upper $95 \%$ confidence interval. Bold numbers represent comparisons that were significantly different from the elephant values; red numbers represent comparisons that were significantly different from the clouded leopard values. See text for further explanation. 
of any conventional statistical analysis that requires the use of covariances (e.g., principal components, ordinary least squares regression, and even Pearson correlations) is that error terms are uncorrelated (Williams et al., 2013). This requirement is not met when, between variables, there are relationships that are due to multiple sources. Such is the case in neuroanatomy, where animals have both a genetic and a socio-developmental background. The confluence of these phylogenetic and ontogenetic factors shape the underlying neuronal morphology of an individual animal as well as the common characteristics of conspecifics. Consequently, it is not possible to evaluate the null hypothesis in the present study with a conventional statistical test (Williams et al., 2013). Fifth, sample size constrains the ability to use statistics to test differences between members of a species or between species themselves. One common rule for designs with nested levels within groups calls for a minimum of 30 units at each level of measurement (Bell et al., 2008). A study of dendritic characteristics between two species would require 30 specimens from each, with a sample of enough neurons to generate 30 randomly selected neurons, from which 30 randomly chosen dendritic trees would be selected, and so on. It is highly unlikely that a researcher would have access to 60 animals divided equally between two species, or the ability to stain and trace a minimum of $1800 \mathrm{com}$ plete dendritic trees. Sixth, inferential statistical methods such as $t$-tests and ANOVAs, with their standard errors, test statistics, and $p$-values require that study samples be randomly selected (Friedman, 1991; Berk and Freedman, 2003). In comparative neuromorphology research, there are no random samples where each unit analyzed has an equal probability of being selected. All samples in such studies are those of convenience, and may not represent any definable population larger than itself (Freedman, 2004).

The present study therefore sought to employ an analytic method that could provide solid evidence of the ability to differentiate species from dendritic measures despite the variability introduced by factors such as differential brain fixation and neuronal staining. Moreover, the analyses had to make these predictions despite violations of the uncorrelated error, random sample, and sample size requirements, which invalidate the use of any conventional statistical tests (e.g., F-tests or principal components). Thus, we were limited to nonparametric techniques that make no assumptions about distributional, correlational, random sampling, or other requirements. The technique chosen is referred to as MARS, MARSplines, or Multivariate Adaptive Regression Splines (Statistica, release 12; StatSoft Inc, Austin, TX; Friedman, 1991; Hastie et al., 2009). The MARSplines technique is appropriate because it does not assume or impose any restrictions or conditions for the differentiation of species with dendritic measures, and it can create useful models even in quite difficult situations similar to those faced in quantitative neuromorphology (http://sdn.statsoft.com/STATISTICAVisualBasic.aspx?page= category\&item $=$ modules $\% 3$ AStatistics $\% 3$ ASTAMARSplines).

Further, examination of the mathematics of MARSplines demonstrates that there are no distributional assumptions or sample size requirements for the $r^{2}$ statistic it generates. For these reasons, the MARSplines analysis was employed in the present study to explore potential species differences in dendritic measures.
Table 3 | Matrix of Spearman's rho correlations between cerebellar volume and dendritic measures (and soma size) across all neuron types. $^{a}$

\begin{tabular}{lccccc}
\hline $\begin{array}{l}\text { Dependent } \\
\text { measure }\end{array}$ & \multicolumn{5}{c}{ Neuron type } \\
\cline { 2 - 6 } Vol & Stellate & Basket & Lugaro & Golgi & Granule \\
\hline TDL & $0.574^{* *}$ & $0.512^{* *}$ & $0.554^{* *}$ & $0.407^{* *}$ & 0.233 \\
& $0.507^{* *}$ & $0.393^{* *}$ & $0.384^{* *}$ & 0.174 & 0.233 \\
\hline MSL & $0.511^{* *}$ & $0.520^{* *}$ & $0.353^{*}$ & $0.343^{*}$ & 0.007 \\
& $0.434^{* *}$ & $0.422^{* *}$ & 0.156 & 0.095 & 0.007 \\
\hline DSC & $0.583^{* *}$ & $0.456^{* *}$ & 0.177 & 0.088 & 0.026 \\
& $0.582^{* *}$ & $0.406^{* *}$ & 0.054 & 0.210 & 0.026 \\
\hline Tor & 0.175 & $0.331^{* *}$ & $0.349 *$ & $0.301 *$ & 0.026 \\
& 0.048 & 0.223 & 0.189 & 0.051 & 0.026 \\
\hline Soma size & $0.242^{*}$ & 0.199 & 0.060 & $0.537^{* *}$ & -0.021 \\
& 0.180 & 0.153 & 0.094 & $0.377^{*}$ & -0.021 \\
\hline
\end{tabular}

${ }^{a}$ Correlations in black font are for all eight species ( $N=317$ neurons); correlations in red font are for all species except the elephant ( $N=297$ neurons). Note the reduction in the magnitude of all correlations when the elephant is removed from the analysis. ${ }^{*} p=0.05 ;{ }^{*} p=0.01$.

\section{RESULTS \\ OVERVIEW}

In terms of gross anatomy across the sampled species, there was a 64 -fold variation in brain mass (from an average of $78 \mathrm{~g}$ in the leopards to an average of $4990 \mathrm{~g}$ in the elephants) and a 103fold variation in cerebellar volume (from an average of $9 \mathrm{ml}$ in the leopards and an average of $924 \mathrm{ml}$ in the elephants). The larger variation in cerebellar volume appears to be a result of a disproportionately large cerebellum in the elephant (Maseko et al., 2012b). A Spearman's rho correlation between brain mass and cerebellar volume revealed a strong positive relationship $\left[r_{(13)}=0.977, p=0.01\right]$. Cerebellar volume averaged $13.6 \pm$ $3.1 \%$ of total brain mass, with the following breakdown: whale (18.6\%), elephant $(18.5 \%)$, giraffe $(13.6 \%)$, tiger $(13.8 \%)$, manatee $(13.4 \%)$, leopard (11.1\%), chimpanzee (10.4\%), and human $(9.3 \%)$. Dendritic measures and soma size also tended to increase with cerebellar volume for most neuronal types, particularly in terms of dendritic Vol and TDL (Table 3). However, removing the elephant data resulted in a $32 \%$ decrease in the magnitude of these correlations and a reduction in the number of significant correlations from 17 to 8 (Table 3), suggesting that the elephant measures were skewing overall results.

Histologically, there was considerable variation in the Golgi stain across species. Each impregnation was nevertheless of sufficient quality to allow for adequate quantification of selected neurons (Figures 2-7). The expected trilaminate architecture of cerebellar cortex was present in all species. The molecular layer, similar to supragranular layers in the cerebral neocortex (Jacobs et al., 1997, 2001), tended to stain better (i.e., exhibited a clearer 

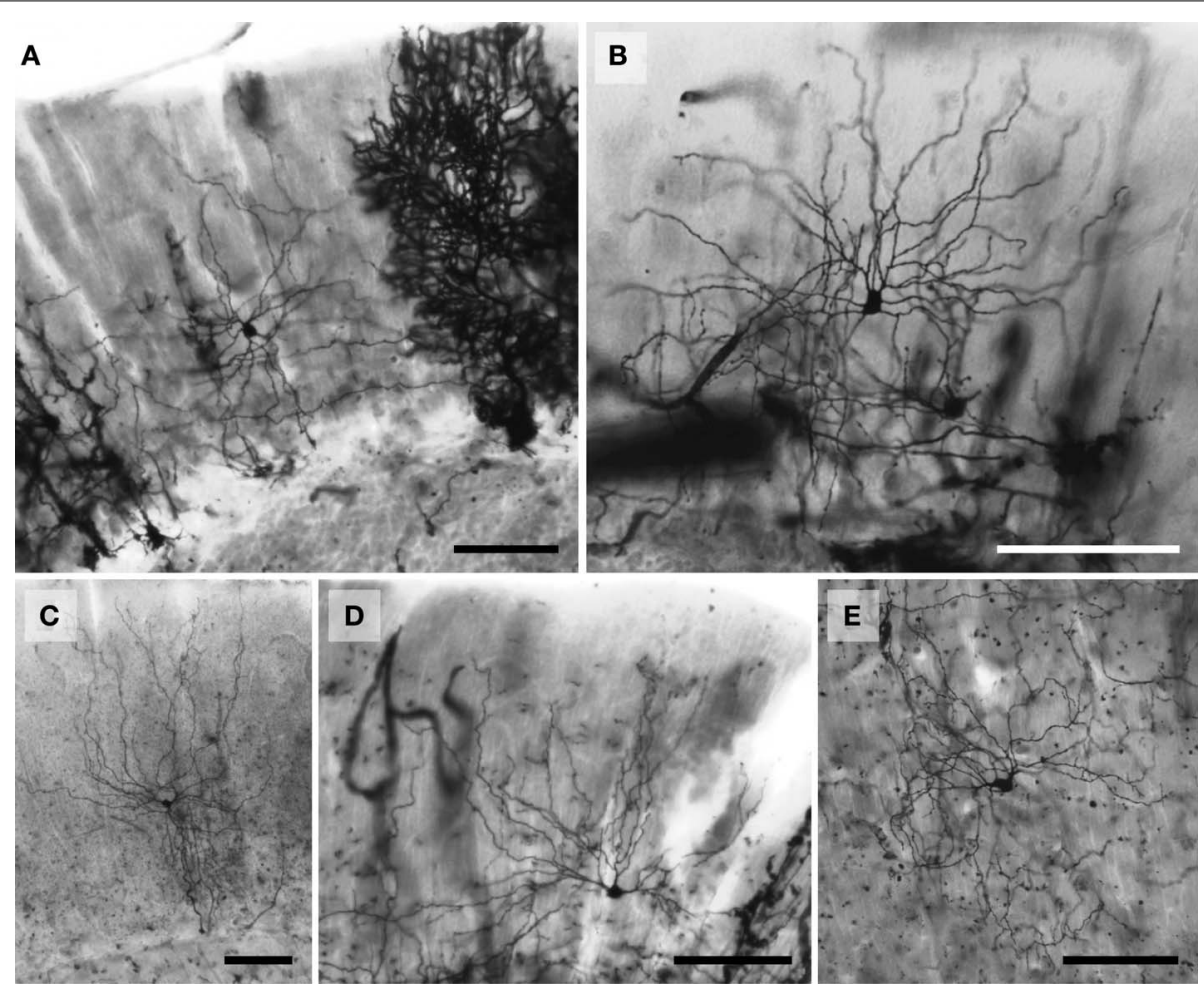

FIGURE 2 | Photomicrographs of Golgi-stained stellate neurons. (A) Florida manatee; (B) chimpanzee (see also Figure 110); (C) human (see also Figure 11D); (D) giraffe; and (E) African elephant (see also Figure 8C). Scale bars: $100 \mu \mathrm{m}$.

background with less obstructed, more complete neurons) than the deeper granule cell layer, which allowed more molecular than granular layer inhibitory interneurons to be traced (Table 1). A Spearman's rho correlation indicated a significant $(p=0.01)$ positive relationship between soma size and all dendritic measures in the total sample [Vol: $r_{(317)}=0.826$; TDL: $r_{(317)}=0.497$; MSL: $r_{(317)}=0.647$; DSC: $r_{(317)}=0.253$ ] except Tor, which was negative $\left[r_{(317)}=-0.285\right]$. In terms of morphology, traced neurons tended to be similar across all species. When comparing across all neuron types, molecular layer interneurons consistently fell in the middle of all dendritic measures. The largest neurons traced were the Lugaro neurons (dendritic Vol ranged from $1454 \mu \mathrm{m}^{3}$ in the chimpanzee to $37,396 \mu \mathrm{m}^{3}$ in the elephant) and the Golgi neurons (TDL ranged from $1456 \mu \mathrm{m}$ in the chimpanzee to $5664 \mu \mathrm{m}$ in the elephant). Lugaro neurons also tended to have the longest MSL values (ranging from $41 \mu \mathrm{m}$ in the giraffe to $109 \mu \mathrm{m}$ in the tiger) whereas Golgi neurons tended to have the highest DSC values (ranging from 30 in the leopard to 143 in the elephant). Granule neurons exhibited the lowest values for every measure except Tor, which obtained its highest value in granule neurons (ranging from 1.17 in the humpback whale to 1.51 in the human).

Sample tracings of neuronal types for each species are provided in Figures 8-11. Mean values of selected dependent measures (i.e., Vol, TDL, MSL, DSC) for each neuronal type across species are presented in Figure 12. Although the graphs in Figure 12 illustrate mean values, only the ranges across species are used in the text below for these dendritic measures because (1) there is asymmetric variation in the dependent measures across and within species and neuronal types, and (2) the extremely large values exhibited by the elephant for most of the dendritic measures distort the overall means. The morphological characteristics of these neuronal types are addressed in detail below, followed by the results from interspecies comparisons of dendritic measures.

\section{MOLECULAR LAYER}

Stellate neurons (Figure 2) were the most superficial neurons traced $\left(\right.$ Mean $\left._{\text {soma depth }}=2.3 \pm 108 \mu \mathrm{m}\right)$. Their round or ovoid somata were smaller than all other neurons except granule cells, with a 2.13-fold range in size across species (chimpanzee $=86 \mu \mathrm{m}^{2}<$ human $<$ whale $<$ giraffe $<$ leopard $<$ tiger $<$ manatee $<$ elephant $=183 \mu \mathrm{m}^{2}$ ). Sample tracings of stellate neurons are provided for each species: African elephant (Figures 8A-C), Florida manatee (Figures 8M-Q), Siberian tiger (Figures 9A-C), clouded leopard (Figures 9S-V), humpback whale (Figures 10F-H), giraffe (Figures 10R-T), human (Figures 11D-F), and chimpanzee (Figures 11P-R). Morphologically, stellate neurons exhibited twisting dendrites that frequently approached the pial surface. Some appeared bipolar (Figure 10S) whereas others had multiple dendrites radiating in all directions (Figures 8A, 11D,P). Stellate neurons had 4.1 primary branches per neuron (ranging from 2.8 in the tiger to 5.6 in the elephant) with a dendritic plexus that generally appeared more complex in the human and elephant than in other species. 


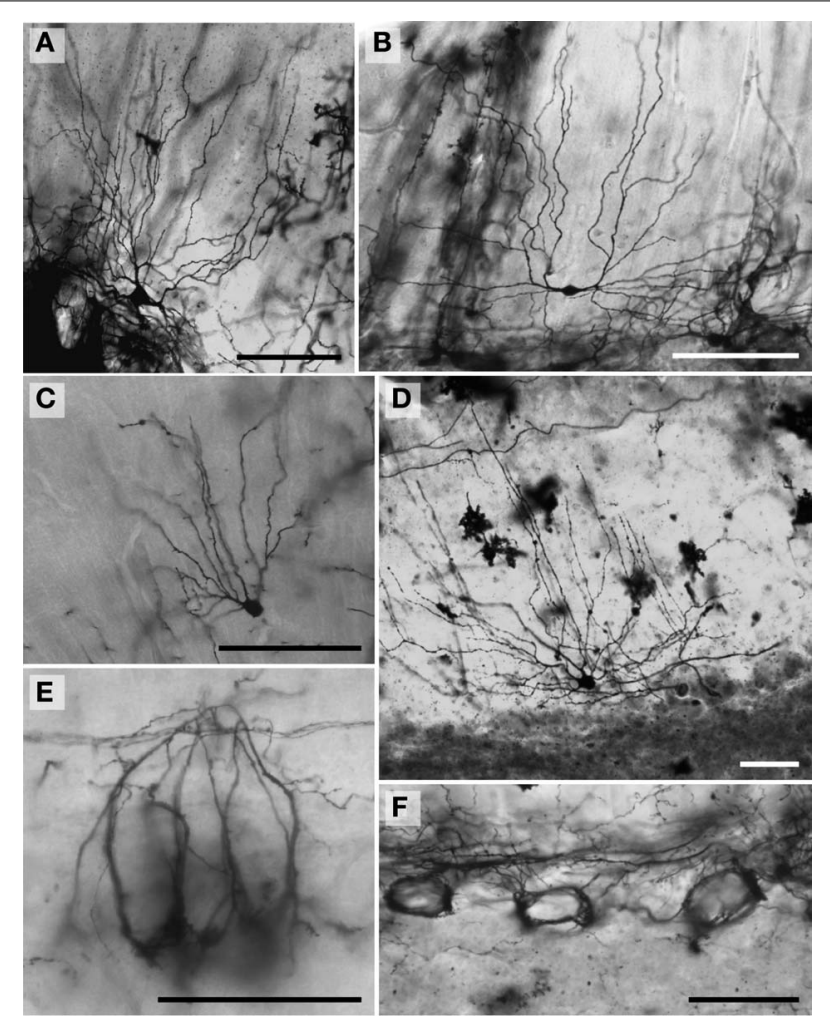

FIGURE 3 | Photomicrographs of Golgi-stained basket neurons. (A) Humpback whale; (B) chimpanzee; (C) giraffe; and (D) African elephant. The pericellular baskets encapsulating Purkinje cell bodies (unstained) are represented in (E) Siberian tiger and (F) humpback whale. Scale bars: $100 \mu \mathrm{m}$.

Quantitatively, dendritic measures varied considerably, although they tended to be greater for the elephant, particularly for dendritic Vol and TDL (Figures 12A-D). Variation in each dendritic measure for stellate neurons across species was as follows: Vol $=$ 14.63-fold, TDL $=4.02$-fold, $\mathrm{MSL}=2.14$-fold, $\mathrm{DSC}=3.56$-fold, and Tor $=1.07$-fold.

Basket neurons (Figure 3) were located in the lower third of the molecular layer $\left(\right.$ Mean $\left._{\text {soma depth }}=350 \pm 134 \mu \mathrm{m}\right)$. Their typically ovoid somata were larger than observed in stellate neurons, with a 3.86-fold difference in size across species (human $=113 \mu \mathrm{m}^{2}<$ chimpanzee $<$ leopard $<$ tiger $<$ whale $<$ giraffe $<$ manatee $<$ elephant $\left.=444 \mu \mathrm{m}^{2}\right)$. Sample tracings of basket neurons are provided for each species: African elephant (Figures 8D,E), Florida manatee (Figures 8R-V), Siberian tiger (Figures 9I-K), clouded leopard (Figures 9W-Y), humpback whale (Figures 10A-E), giraffe (Figures 10U-X), human (Figures 11A-C), and chimpanzee (Figures 11S-V). Morphologically, basket neurons were usually, but not always (Figures 8D, 11C), characterized by dendritic branches that extended laterally from the soma, travelling horizontally a short distance before curving toward the pial surface in a typical sea-fan shape (Figures 8E, 9X, 10D, 11S). Axons were visible in some neurons, allowing them to be traced over distances of several $100 \mu \mathrm{m}$ (Figures 8R, 11C,U). These axons travelled transversely above the Purkinje cell layer and were sometimes observed to terminate in multiple pericellular nests (Figure 3F) with paintbrush tips (Figure 3E; Ramón y Cajal, 1909, 1911) around the somata of Purkinje cells. Basket neurons had an average of 4.1 primary dendrites (ranging from 3.0 in the whale to 6.1 in the manatee). As with stellate neurons, they appeared more dendritically complex in the human and elephant relative to the other species. Quantitatively, there was considerable variation among species, with the elephant generally exhibiting the largest dendritic values (Figures 12E-H). Ranges of variation in each dendritic measure for basket neurons across species were as follows: $\mathrm{Vol}=13.51$-fold, $\mathrm{TDL}=4.83$-fold, $\mathrm{MSL}=1.72$-fold, DSC $=3.10$-fold, and Tor $=1.07$-fold.

\section{PURKINJE CELL LAYER}

Purkinje neurons were not quantified in the present sample because their dendritic complexity precluded (accurate) tracings; in fact, the distal dendritic plexuses in many of these neurons were completely black under the 60x objective. Nevertheless, sample photomicrographs (Figure 4) illustrate large, piriform somata from which the complex, prototypical two-dimensional dendritic plexus ascended throughout the molecular layer. These appeared morphologically similar across all species except the humpback whale (Figure 4C), where tertiary dendritic branches tended to ascend to the pial surface in straight, unbending manner. As such, the main dendritic branches of the humpback Purkinje neuron are much less convoluted than observed in the other species. This morphological difference is particularly clear when comparing the skeletal tracings of the humpback whale Purkinje neurons (Figures 4F,G) to those of the giraffe (Figures 4H,I), the other cetartiodactyl in the current study.

\section{GRANULE CELL LAYER}

Lugaro neurons (Figure 5) were usually located superficially in the granule cell layer immediately below the Purkinje cell bodies $\left(\mathrm{Mean}_{\text {soma depth }}=543 \pm 179 \mu \mathrm{m}\right)$. Those Lugaro neurons located between Purkinje cell and granule cell layers usually possessed fusiform somata (e.g., Figures $8 \mathrm{~K}, 10 \mathrm{~K}, 11 \mathrm{Z}, \mathrm{AA}$ ); those deeper in the granule cell layer were more likely to possess triangular somata. In terms of soma size, these were the largest observed in the present sample, with a 5.01-fold difference in size across species (manatee $=270 \mu \mathrm{m}^{2}<$ chimpanzee and giraffe $<$ human $<$ leopard $<$ tiger $<$ whale $<$ elephant $=$ $\left.1354 \mu \mathrm{m}^{2}\right)$. There was an average of 3.9 primary dendrites per neuron (ranging from 3.2 in the tiger to 5.3 in the leopard). Those neurons with fusiform somata tended to be horizontally oriented in the parasagittal plane with dendrites that branched little and extended over several $100 \mu \mathrm{m}$ (Figures 8K, 9L, 10J,K, 11G). Those with more triangular shaped somata were oriented in various directions, including perpendicular to the Purkinje cell layer, and were particularly common in the elephant (Figures 8J,K, $\mathbf{9 N}, \mathbf{1 1 Y}$ ). In contrast to other cerebellar neurons, which tended to be relatively uniform in appearance, the Lugaro neurons were more morphologically diverse, as indicated in the tracings for each species: African elephant (Figures 8I-L), Florida manatee (Figure 8W), Siberian tiger (Figures 9 L-N), clouded leopard (Figures 9Z-AA), humpback whale (Figures 10I-L), giraffe 


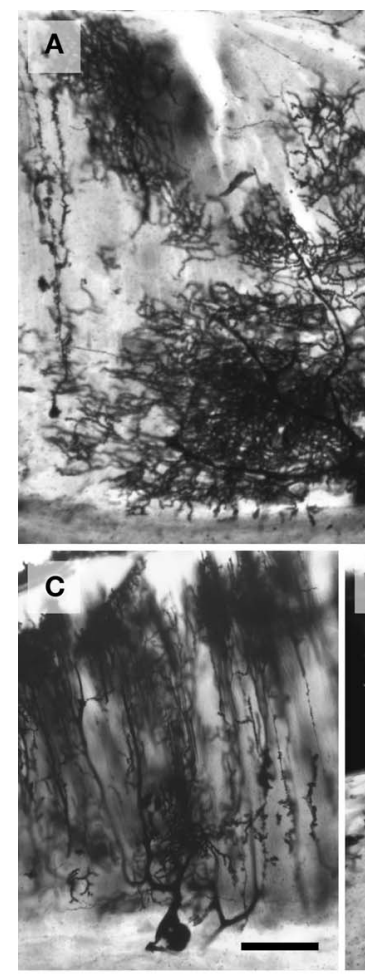

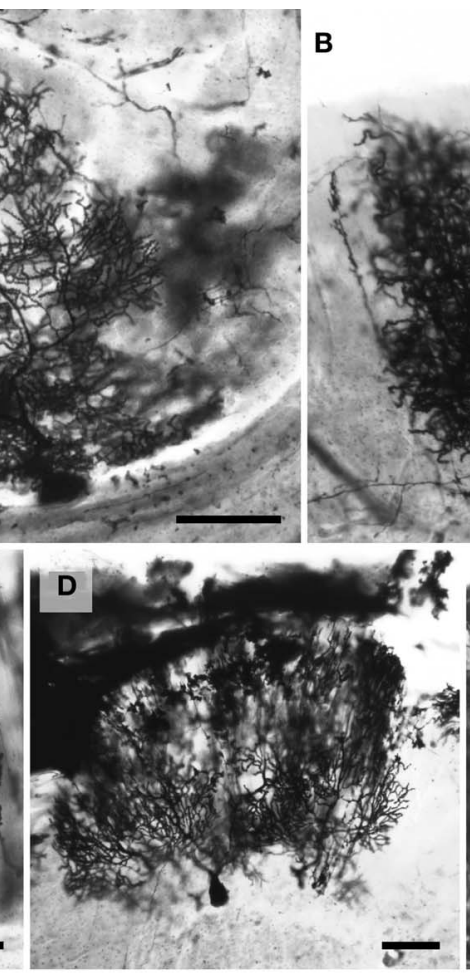

$\mathbf{G}$

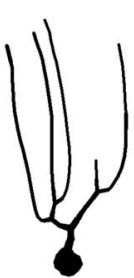

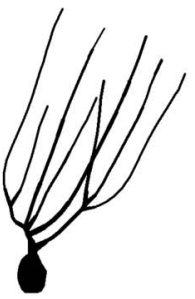

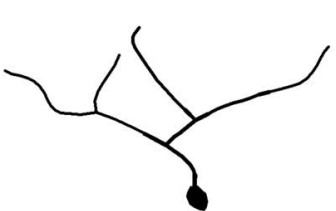

FIGURE 4 | Photomicrographs of Golgi-stained Purkinje neurons. (A) African elephant; (B) human; (C) humpback whale; (D) Florida manatee; and (E) giraffe. Note the large similarity between the neurons of all species except the humpback whale, where the Purkinje neuron appears to have more vertically oriented tertiary branches. Sample tracings of the main dendritic branches illustrating this morphological difference are provided for the humpback whale $\mathbf{( F , G ) , ~ w h i c h ~ d i f f e r s ~ s u b s t a n t i a l l y ~ e v e n ~ f r o m ~ t h e ~ o t h e r ~}$ cetartiodactyl in the study, the giraffe $\mathbf{( H , I )}$. Scale bars: $100 \mu \mathrm{m}$.
(Figures 10Y-AA), human (Figures 11G-I), and chimpanzee (Figure 11W-AA). Quantitatively, Lugaro neurons were disproportionately larger in the elephant than in any of the other species, particularly in terms of dendritic Vol (Figures 12I-L). Variation in each dendritic measure for Lugaro neurons across species was as follows: $\mathrm{Vol}=25.72$-fold, $\mathrm{TDL}=3.36$-fold, $\mathrm{MSL}=2.66$-fold, DSC $=3.44$-fold, and Tor $=1.08$-fold.

Golgi neurons (Figure 6) were also usually located superficially in the granule cell layer, although some were much deeper $\left(\mathrm{Mean}_{\text {soma depth }}=512 \pm 175 \mu \mathrm{m}\right)$. They had irregular stellate, triangular, or polygonal somata, with a 3.05-fold difference in size across species (leopard $=242 \mu \mathrm{m}^{2}<$ chimpanzee $<$ human $<$ tiger $<$ whale $<$ giraffe $<$ elephant $\left.=737 \mu \mathrm{m}^{2}\right)$. With an average of 6.5 primary dendrites per neuron (ranging from 5.3 in the whale to 8.2 in the elephant), they exhibited the highest number of primary dendrites in the present sample. These dendrites radiated relatively thick branches in all directions, forming a characteristic three-dimensional spherical field, as illustrated in Neurolucida tracings: African elephant (Figures 8F-H), Siberian tiger (Figures 9D-H), clouded leopard (Figures 9BB,CC), humpback whale (Figures 10P,Q), giraffe (Figures 10BB-EE), human (Figures 11J-L), chimpanzee (Figures 11GG-JJ). Quantitatively, as with most other neurons in the current sample, these achieved their greatest extent in the elephant (Figures 12M-P). Variation in each dendritic measure for Golgi neurons across species (except the manatee) was as follows: $\mathrm{Vol}=12.94$-fold, TDL $=3.89$-fold, $\mathrm{MSL}=1.76$-fold, DSC $=4.77$-fold, and Tor $=1.07$-fold.

Granule neurons (Figure 7) were, on average, the most deeply located of all traced neurons $\left(\right.$ Mean $\left._{\text {soma depth }}=615 \pm 181 \mu \mathrm{m}\right)$, and the smallest, with a 1.36-fold difference in size across species (human $=50 \mu \mathrm{m}^{2}<$ leopard $<$ chimpanzee $<$ whale and tiger $<$ giraffe $\left.=68 \mu \mathrm{m}^{2}\right)$. These were characterized by small, round cell bodies from which an average of 3.5 short 


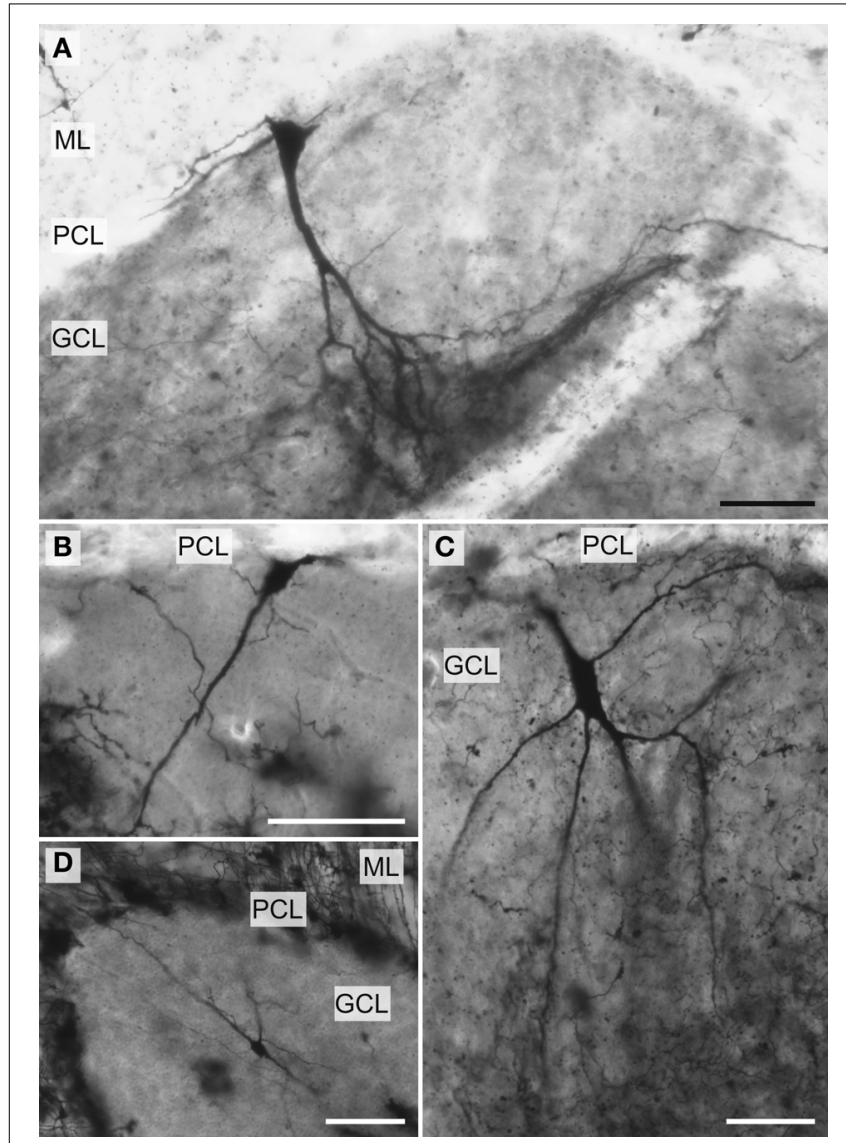

FIGURE 5 | Photomicrographs of Golgi-stained Lugaro neurons. (A, see also Figure $\mathbf{8 J}$ ) and (C, see also Figure $\mathbf{8 L}$ ) African elephant; in these two neurons, note the bouquet shaped dendritic arbor in (A) and the more solitary, unbranched dendritic arbor in (C), with both descending to the underlying white matter. The (B) humpback whale (see also Figure 10L) and (D) chimpanzee also have predominantly unbranched dendritic trees. $\mathrm{ML}$, molecular layer; PCL, Purkinje cell layer; GCL, granule cell layer. Scale bars: $100 \mu \mathrm{m}$

dendrites emerged (ranging from 3.0 in the human to 3.8 in the giraffe, whale, and tiger), each terminating in gnarled, claw-like inflorescences. Axons, when visible, tended to ascend immediately toward the molecular layer (Figures 9Q, 10FF, $\mathbf{1 1} \mathbf{M}, \mathbf{B B}, \mathbf{C C})$. Across species, there was little qualitative variation in granule neuron morphology: Siberian tiger (Figures 90-R), humpback whale (Figures 10M-O), giraffe (Figures 10FF-HH), human (Figures 11M-O), and chimpanzee (Figures 11BB-FF). Quantitatively, granule neurons had the lowest median values of all dendritic measures except Tor, for which they exhibited the highest values (Figures 12Q-T). Variations in each dendritic measure for granule neurons across species (except the elephant and manatee) were as follows: $\mathrm{Vol}=5.34$-fold, $\mathrm{TDL}=2.41$-fold, $\mathrm{MSL}=6.40$-fold, DSC $=2.50$-fold, and Tor $=1.29$-fold .

\section{SHOLL ANALYSES}

Several limited observations can be made on the basis of the Sholl analyses (Figure 13). First, the peak in the number of intersections appeared to be around $100 \mu \mathrm{m}$ from the soma
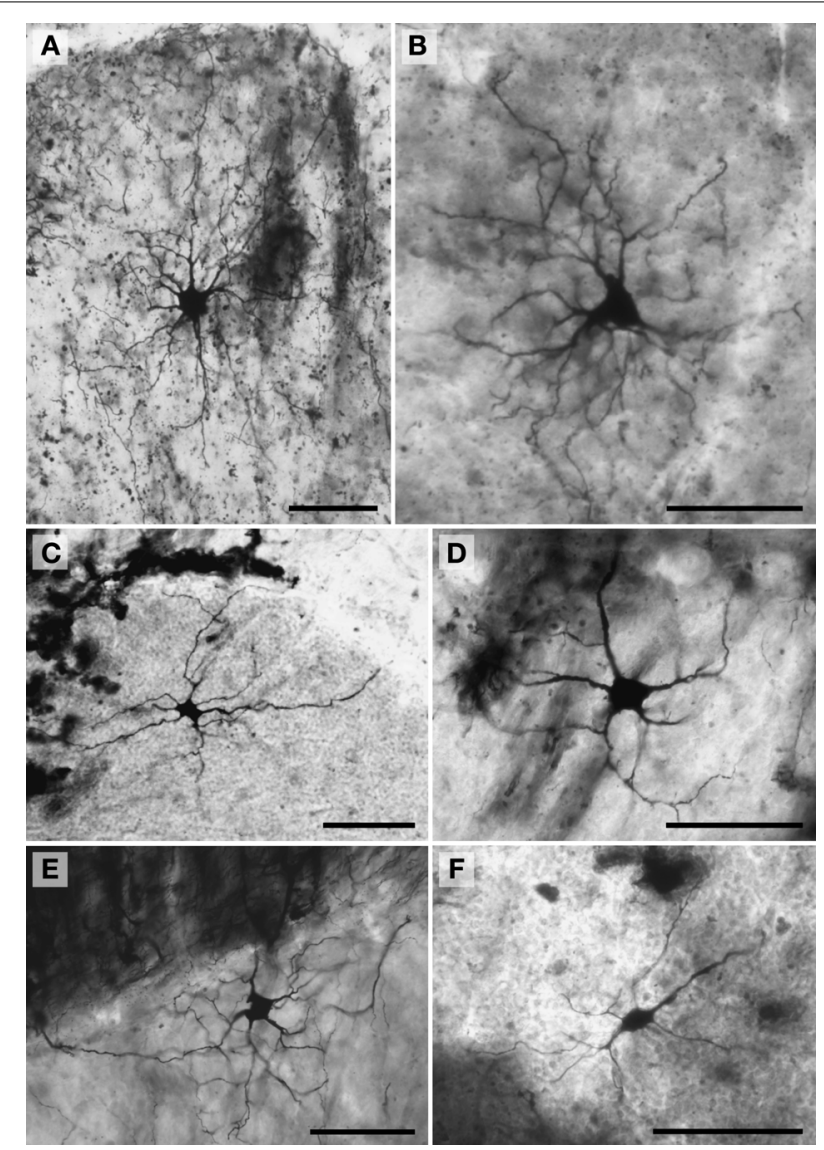

FIGURE 6 | Photomicrographs of Golgi-stained Golgi neurons. (A) and (B, see also Figure 8H) African elephant; (C) chimpanzee (see also

Figure 11GG); (D) Siberian tiger (see also Figure 9E); (E) giraffe (see also Figure 10DD); and (F) clouded leopard (see also Figure 9BB). Scale bars: $100 \mu \mathrm{m}$.

for most neuron types for all species. Second, Lugaro neurons (Figures 13I-L) did not exhibit the same sharp peak in intersections as did other neurons; rather, they were relatively flat in their dendritic envelope, with dendrites that extended great distances from the soma, particularly in humans and elephants. Third, the elephant profile (Figures 13A,E,M) appeared markedly different (i.e., much higher peaks) from other species' profiles. Forth, granule neurons (Figures 13Q-S) had a much lower number of intersections than did other neurons, and exhibited a peak around $25 \mu \mathrm{m}$ from the soma.

\section{INFERENTIAL STATISTICAL ANALYSES ACROSS SPECIES}

To examine species differences in dendritic measures, analyses proceeded with a third order MARSplines differentiation of species using TDL, MSL, DSC, Vol, Tor, and soma size. In brief, the procedure tested the dendritic measures and soma size of each neuron to assess if it could be identified as belonging to a particular species. Eight binary variables were created, one for each species. The analysis proceeded by utilizing a MARSplines model to test the hypothesis that species could be differentiated from each other based on just dendritic measures and soma size. As an 


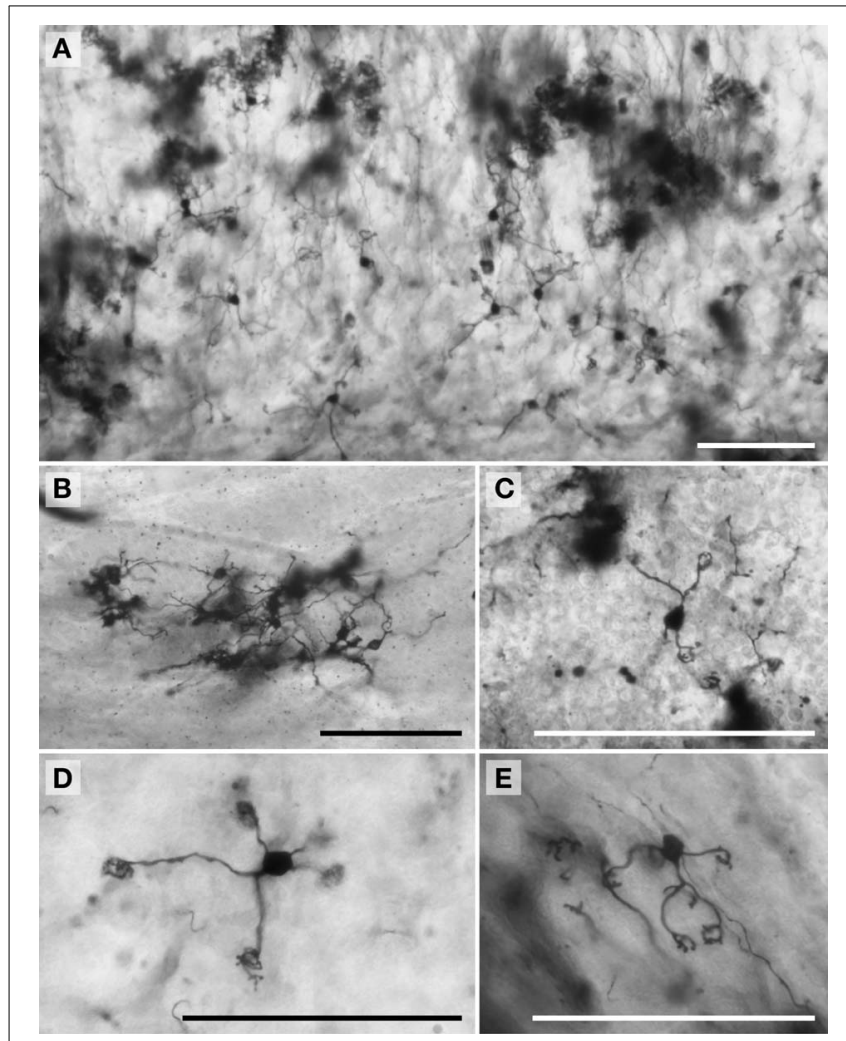

FIGURE 7 | Photomicrographs of Golgi-stained granule neurons. (A) Siberian tiger; (B) humpback whale; (C) chimpanzee; (D) Siberian tiger; and (E) giraffe. Scale bars: $100 \mu \mathrm{m}$.

example, to test the hypothesis with giraffes, a new attribute called giraffe was created, and it took a value 1 when the dendritic measures came from a giraffe neuron and a 0 when they did not. Eight of these binary (1/0) attributes were created, one for each species. Each of the 317 rows of neuronal data was coded with eight 1/0 attributes. For example, there was a row for a giraffe neuron with the eight new species attributes arranged in alphabetical order (chimpanzee, clouded leopard, elephant, giraffe, human, humpback whale, manatee, tiger); then, these attributes were coded 0 , $0,1,0,0,0,0,0$, respectively. Next, the MARSplines analysis was used with the giraffe attribute as the dependent measure and the dendritic measurements as the independent measures to test the null hypothesis by comparing neuronal measures contributed by the giraffe to those contributed by the other seven species. In the present study, the null hypothesis was that there was no relationship between the dendritic measures and species, or, in other words, that the dendritic measures for a given neuron could not be assigned reliably to a given species. The null hypothesis was rejected at either $p<0.10$ on the $F$-test or a correct prediction $(\geq 0.90)$ about whether a neuron did or did not belong to a particular species. Results were represented as counts (i.e., number of neurons that successfully differentiated a given species from all the other species) and percentages of correct and incorrect species assignments, an $r^{2}$ statistic, and an $F$ statistic. $F$ statistics were produced using each binary species attribute as the categorical or class measure and the "is or isn't" as the target species score estimated from the MARSplines. The estimated scores were continuous, and the cut point for whether or not the estimated value was the species under analysis or some other species was 0.5 . This cut point was used in calculating the percentage of correct predictions. That is, if giraffes were being evaluated, the observed value of the giraffe $1 / 0$ attribute would have been 1 and if the estimated value of giraffe was $\geq 0.5$, we would conclude that the MARSpline equation correctly differentiated giraffes from all other species in that row of data.

The null hypothesis of no relationship between dendritic measures and species was rejected for all species because all $F$ statistics were significant at $p \leq 0.01$ : elephant $\left[F_{(1,253)}=8097.30\right.$, $\left.r^{2}=0.967\right]$, manatee $\left[F_{(1,267)}=334.33, r^{2}=0.526\right]$, tiger $\left[F_{(1,315)}=137.93, r^{2}=0.265\right]$, leopard $\left[F_{(1,315)}=110.63, r^{2}=\right.$ $0.260]$, whale $\left[F_{(1,315)}=373.76, r^{2}=0.208\right]$, giraffe $\left[F_{(1,315)}=\right.$ $\left.290.12, r^{2}=0.448\right]$, human $\left[F_{(1,315)}=196.05, r^{2}=0.349\right]$, and chimpanzee $\left[F_{(1,315)}=720.62, r^{2}=0.443\right]$. Further, as noted in the correct-incorrect confusion matrices (Table 4), the percentage of correct predictions for neuronal fit to a particular species ranged from $85.5 \%$ in the chimpanzee to $99.6 \%$ in the elephant. To elaborate, in the elephant, 19 of 20 neurons were correctly identified as belonging to the elephant, and 235 of 235 were correctly identified as not belonging to the elephant (thus, 99.6\%). In the chimpanzee, 63 of 86 neurons were correctly identified as belonging to the chimpanzee, and 208 of 231 were correctly identified as not belonging to the chimpanzee (thus, 85.5\%). What these results indicate is that dendritic measures and soma size were accurate predictors of each species in the current sample because these measures, taken together, allowed neurons to be correctly identified as belonging to a particular species.

Further, the procedure provided the relative importance of each attribute (i.e., dendritic Vol, TDL, soma size, etc.) in determining whether a neuron belonged or did not belong to a particular species (Table 4). These measures indicated the number of times each attribute or predictor was used in the equations testing the null hypothesis for each species. In this framework, for example, an attribute with three appearances in the analysis for a given species would be three times more important to the prediction than an attribute with only one appearance. As noted in Table 4, across all species, dendritic Vol, Tor, and TDL appeared to be the overall most important (i.e., most utilized by the analysis) measures for differentiating each species from the others, whereas DSC was the least important. However, the combination of these variables was unique for each species. For example, in the elephant, TDL (17 appearances) and dendritic Vol (16 appearances) were the most influential measures in species identification; in the chimpanzee, however, soma size (9 appearances) and dendritic Vol (6 appearances) were the most important predictors.

\section{DISCUSSION}

The present study contributes to a limited database of comparative neuroanatomy (Manger et al., 2008) by examining cerebellar neuronal morphology across a wide variety of large brained mammals both qualitatively and quantitatively. Although the current sample exhibited a large range in cerebellar volume, the overall volume fraction of the cerebellum $(13.6 \pm 3.1 \%)$ is consistent 


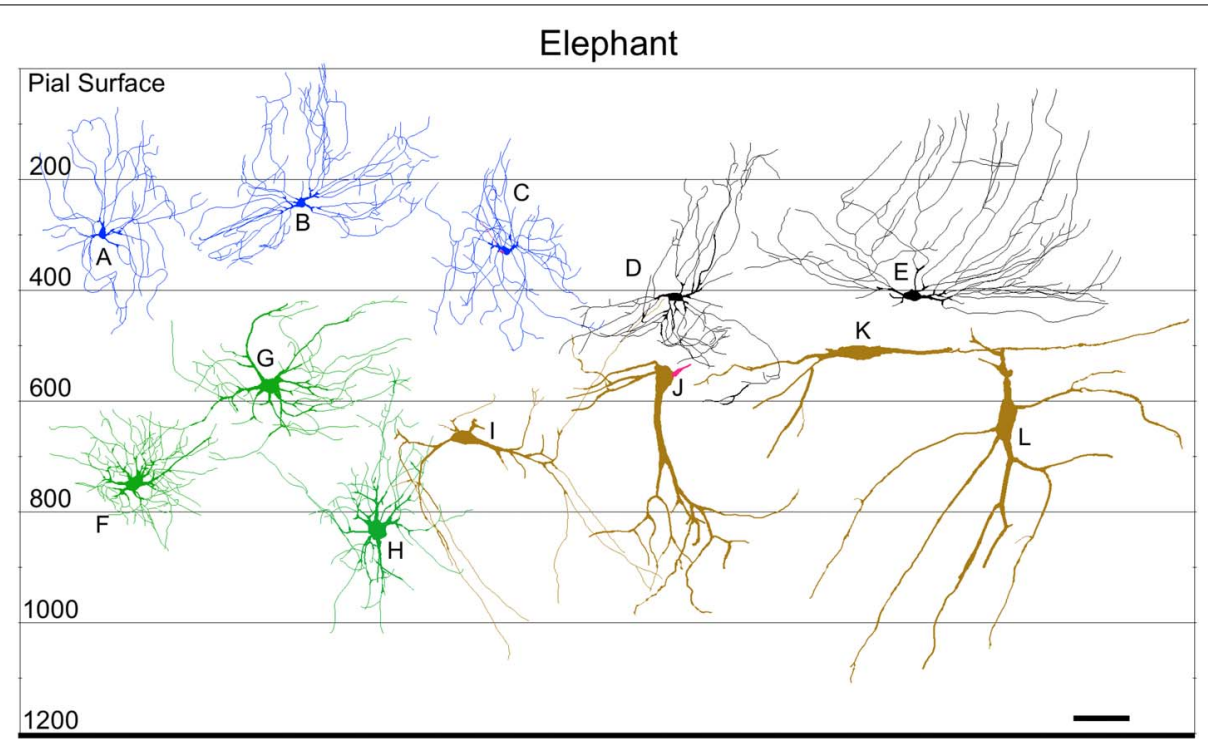

Manatee

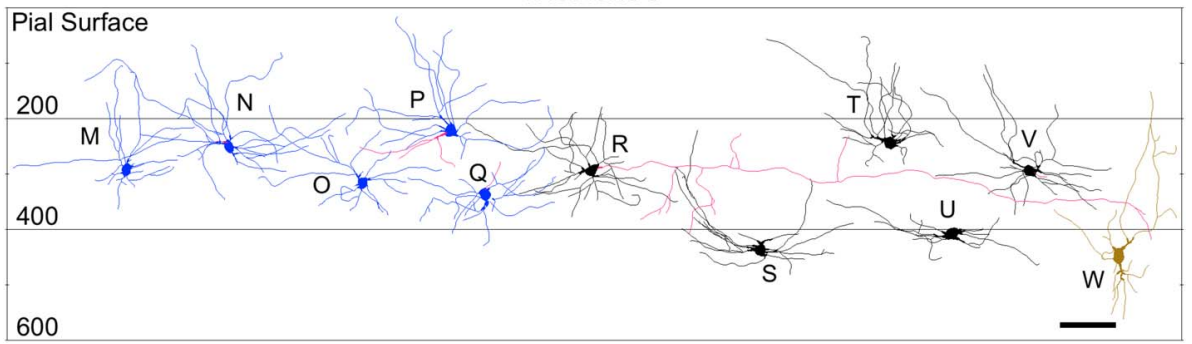

FIGURE 8 | Neurolucida tracings of neurons in the cerebellar cortex of the African elephant (top) and Florida manatee (bottom) indicating relative soma depth from the pial surface (in $\mu \mathbf{m})$. Stellate neurons (A-C; $\mathbf{M}-\mathbf{Q}$ ); basket neurons (D,E; R-V); Lugaro neurons (I-L; W); Golgi neurons (F-H). Scale bar: $100 \mu \mathrm{m}$.

with that reported by Clark et al. (2001) across 9 mammalian taxa, namely $13.5 \pm 2.4 \%$. There was considerable uniformity across species in terms of histology insofar as the cerebellar cortex followed the trilaminate pattern typical of birds and mammals (Ramón y Cajal, 1909, 1911; Sultan and Glickstein, 2007). In terms of morphology, each neuronal type within the cerebellar cortex was generally consistent across the eight species. Quantitatively, however, there was substantial species variation in dependent dendritic measures for each neuronal type, with neurons in the elephant tending to be larger than those in other species for most measures. Finally, inferential analyses detected significant species differences in dendritic measures and soma size.

\section{METHODOLOGICAL CONSIDERATIONS}

General constraints pertaining to Golgi-stained materials have been extensively outlined elsewhere (Jacobs and Scheibel, 2002; Jacobs et al., 2011). These include (1) characteristics of incomplete impregnations (Williams et al., 1978; Braak and Braak, 1985), (2) the effects of post-mortem delay and suboptimal fixation (de Ruiter, 1983; Jacobs and Scheibel, 1993; Jacobs et al., 1993, 2001; Friedland et al., 2006), and (3) the relative merits of the Golgi stain compared to other histological techniques
(Scheibel and Scheibel, 1978; Buell, 1982; Ohm and Diekmann, 1994; Jacobs et al., 1997). Another inherent limitation in Golgi studies is the effect of section thickness on estimations of dendritic extent (Jacobs et al., 1997). Larger neurons (such as those in the elephant) are more affected by sectioning, resulting in an attenuation of dendritic measures. In the present study, this means that actual differences among species are probably larger than the data suggest. Thus, the present dendritic measurements should be seen as representing relative rather than absolute values. To completely eliminate cut dendrites would require (1) tissue sections $\sim 1000 \mu \mathrm{m}$ thick, which would make them completely opaque, or (2) tracing cut dendritic segments across serial sections, a technique that is not accurate or feasible in a Golgi study this extensive, where multiple, overlapping neural processes appear in any given section of tissue. Finally, neurons in the present study were classified based solely on somatodendritic architecture and their relative location within the cerebellar cortex, which is typical for Golgi impregnations. It was not possible to further subcategorize neuron types based on axonal plexi distributions (Bishop, 1993; Lainé and Axelrad, 1996), lipofuscin pigmentation (Braak and Braak, 1983), or immunohistochemistry and neurochemical phenotypes (Lainé and Axelrad, 2002; Simat et al., 2007). 

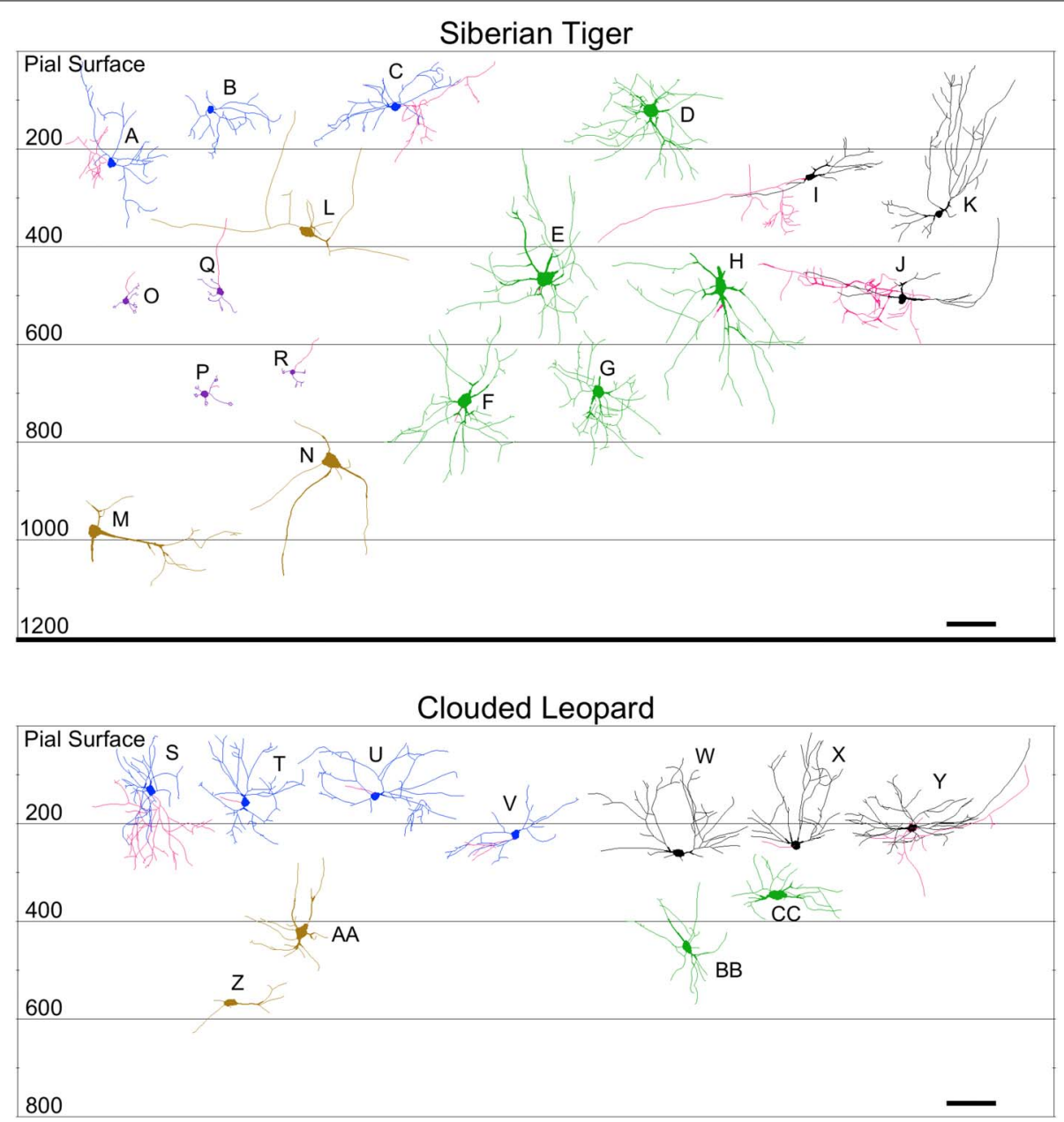

FIGURE 9 | Neurolucida tracings of neurons in the cerebellar cortex of the Siberian tiger (top) and clouded leopard (bottom) indicating relative soma depth from the pial surface (in $\mu \mathrm{m}$ ). Stellate neurons (A-C; S-V): basket neurons (I-K; W-Y); Lugaro neurons (L-N; Z-AA); Golgi neurons (D-H; BB,CC); granule neurons (O-R). Axons, when present, are indicated in red. Scale bar: $100 \mu \mathrm{m}$.

\section{NEURONAL MORPHOLOGY: OUALITATIVE OBSERVATIONS}

In general, neurons in the present sample were similar in morphology to those described in primates (Fox et al., 1967; Rakic, 1972; Braak and Braak, 1983; Mavroudis et al., 2013) and rodents (O'Leary et al., 1968; Chan-Palay and Palay, 1972; Palay and Chan-Palay, 1974). For example, molecular layer interneurons in the present sample had hemielipsoid dendritic systems that greatly resembled those described by Rakic (1972), with deeper neurons having an ascending dendritic plexus, intermediate neurons having a dendritic plexus extending in all directions, and superficial neurons having mostly descending dendritic branches. The axonal ramifications observed in basket neurons were also similar to those described in monkeys (Fox et al., 1967), cats (Bishop, 1993), and rats (Palay and Chan-Palay, 1974). There were, however, a few morphological observations of particular interest. First, the Lugaro neurons in most of the current species resembled those described in the literature (Christ, 1985; Melik-Musyan and Fanardzhyan, 1998, 2004). They also resembled those identified by Braak and Braak (1983) in humans as Type II neurons despite Braak and Braak stating that these types of cells may be displaced basket neurons, a finding disputed by others (Lainé and Axelrad, 1996). In contrast, many Lugaro neurons in the elephant appeared distinctive because of their vertical orientation and idiosyncratic dendritic arrangements. These were unusual in the present sample, although they have been briefly described in the cat (Sahin and Hockfield, 1990; MelikMusyan and Fanardzhyan, 1998) and the duck (O'Leary et al., 1968). Second, most of the traced Golgi neurons resembled those observed in the literature, and are consistent with Braak and Braak's (1983) Type I neuron designation. However, there are some (e.g., Figures 8G, 11K) that resembled Braak and Braak's Type III description (see their Figure 7) insofar as they exhibited, among other characteristics, a very dense dendritic arborization that extended into the molecular layer.

Purkinje neurons were remarkably similar in their basic morphology across most species examined, and were consistent with those described in other species (rat: Roth and Häuser, 2001; Sawada et al., 2010; guinea-pig: Rapp et al., 1994). The notable exception was the Purkinje neuron in the humpback whale, which exhibited straighter, more vertically oriented tertiary dendritic 


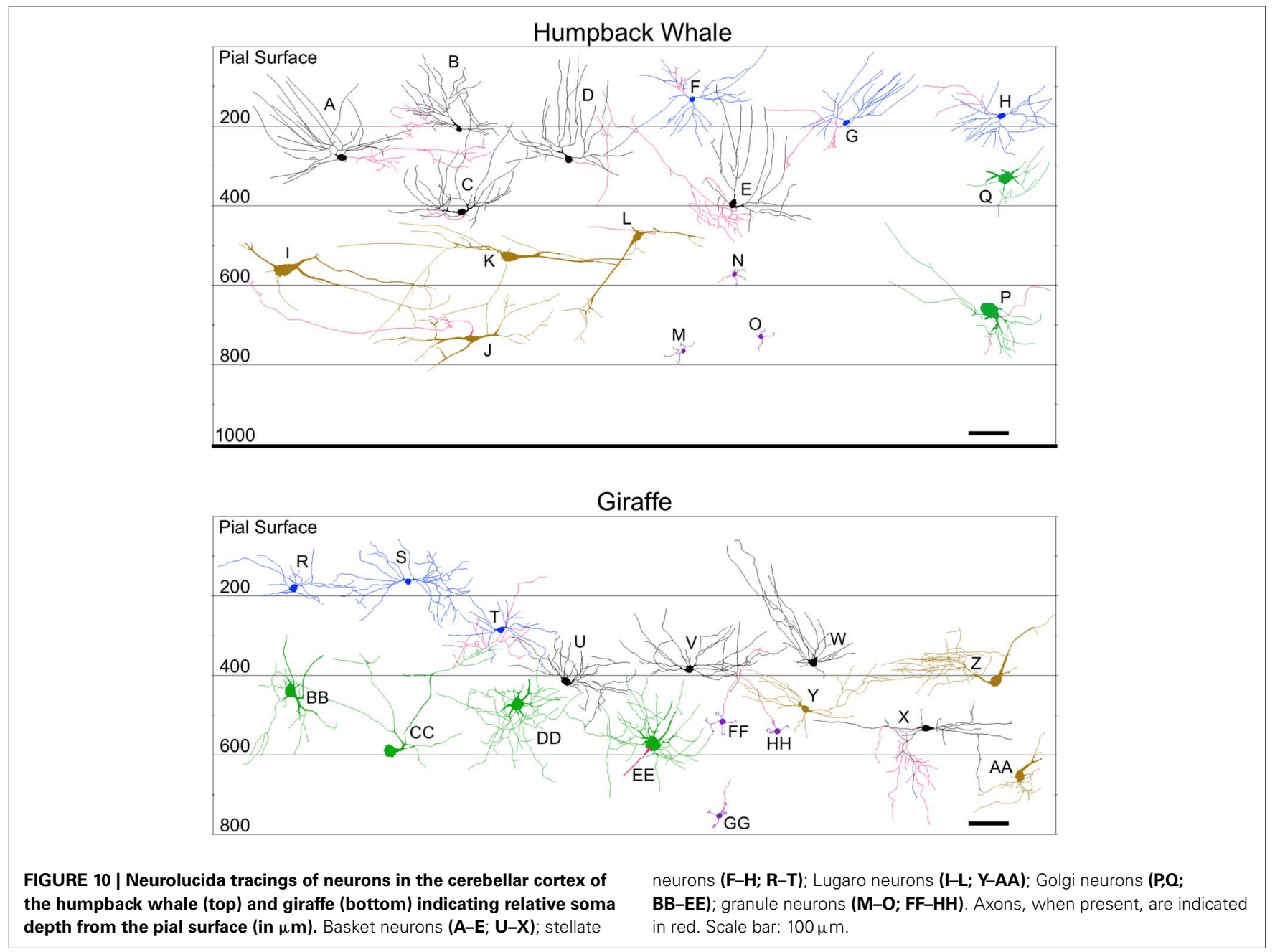

arbors than any of the other species examined. This dendritic pattern differed from the other aquatic mammal in the current study (e.g., the manatee) and from the other cetartiodactyl (e.g., the giraffe). Humpback whale Purkinje neurons actually resembled those observed in mormyrid electric fish (Meek and Nieuwenhuys, 1991; Meek, 1992; Han et al., 2006), with a palisade pattern of relatively unbranched, molecular layer dendritic arbors that extend in parallel to the pial surface (Nieuwenhuys and Nicholson, 1967; Meek and Nieuwenhuys, 1991). Visual observation of our Golgi stains suggests, however, that the humpback whale may have substantially fewer palisade dendrites than the $\sim 50$ noted in mormyrid Purkinje neurons (Meek and Nieuwenhuys, 1991). Direct comparison within cetaceans is problematic because only Adanina (1965) provides any images of Golgi impregnated cerebellar cortical neurons in cetaceans (specifically, Tursiops truncatus and Delphinus delphis). Adanina suggests that there is heterogeneity in the somata of Purkinje neurons, with some being pear-shaped and others being fusiform. Unfortunately, although Purkinje neuron dendritic arbors in the dolphin may be consistent with our observations in the humpback whale, Adanina's impregnation is insufficient for a definitive conclusion. Further research is necessary to confirm whether
Purkinje neurons in mysticetes are morphologically different from those in other cetaceans, or from mammals in general.

\section{NEURONAL MORPHOLOGY: OUANTITATIVE OBSERVATIONS}

With the exception of Purkinje neuron reconstructions and a few measurements of individual dendritic segments, there appear to be very limited quantitative dendritic data on cerebellar cortical neurons (Palay and Chan-Palay, 1974; Braak and Braak, 1983). There is, however, a small number of digital reconstructions of molecular layer interneurons (classical stellate and basket neurons) in the rat based on rapid Golgi impregnations (Sultan and Bower, 1998). Not surprisingly, soma size $\left(69 \mu \mathrm{m}^{2}\right)$ in these rat neurons was smaller than in stellate neurons from all species in the present sample. The number of primary branches (3.1) in the rat was also near the minimum of the current sample (tiger: 2.8 primary branches in stellate neurons; whale: 3.0 primary branches in basket neurons). Similarly, the dendritic length for rat molecular interneurons $(1189 \mu \mathrm{m})$ was shorter than the lowest values for stellate (leopard TDL $=1367 \mu \mathrm{m}$ ) and basket neurons (tiger $\mathrm{TDL}=1285 \mu \mathrm{m}$ ) in the current sample (see Figure 12), although it should be noted that Sultan and Bower (1998) sectioned their tissue at $100 \mu \mathrm{m}$ rather than $120 \mu \mathrm{m}$, which could result in more 

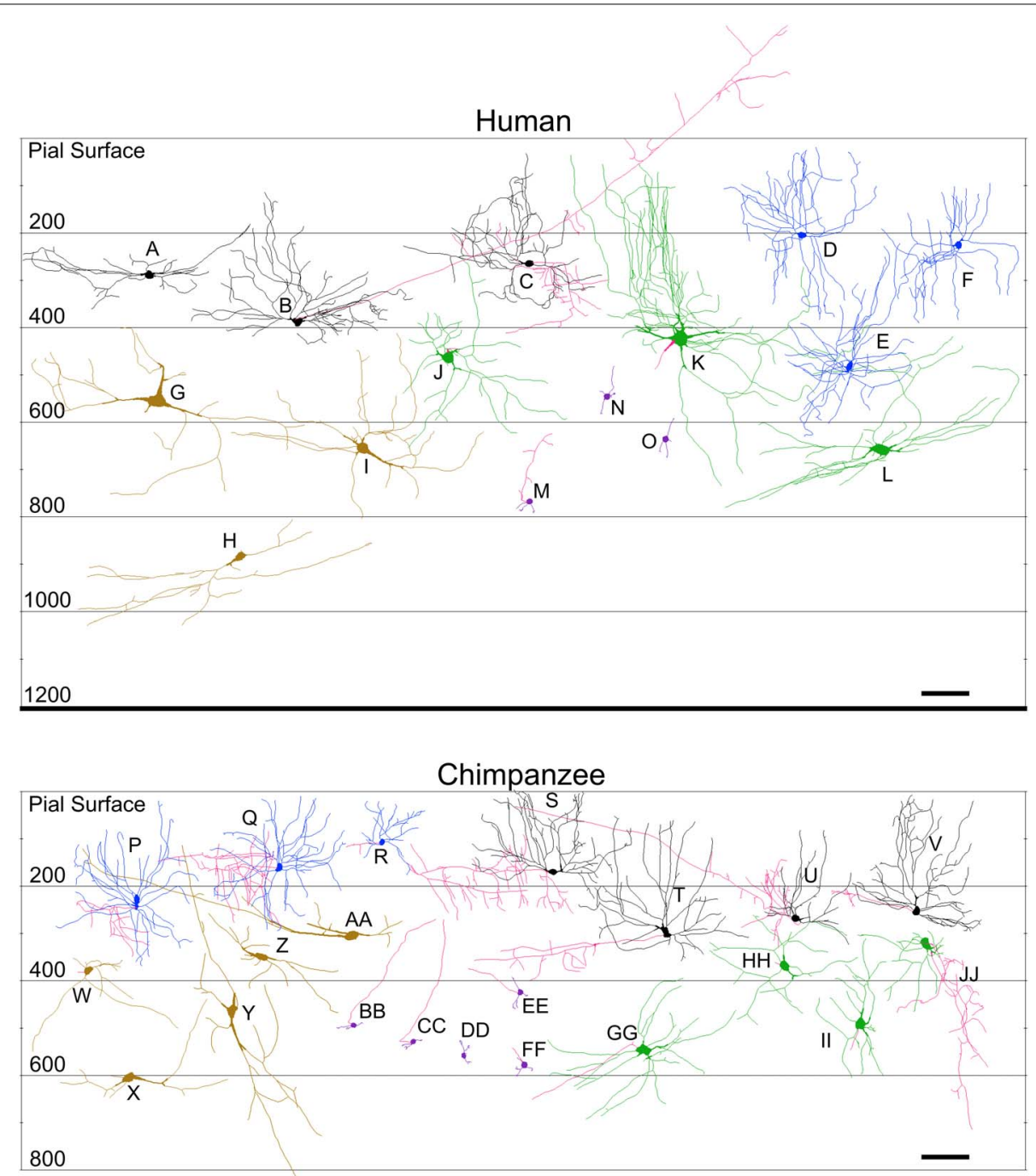

FIGURE 11 | Neurolucida tracings of neurons in the cerebellar cortex of the human (top) and chimpanzee (bottom) indicating relative soma depth from the pial surface (in $\mu \mathbf{m}$ ). Basket neurons (A-C; S-V); stellate neurons (D-F; P-R); Lugaro neurons (G-I; W-AA); Golgi neurons (J-L; GG-JJ); granule neurons (M-O; BB-FF). Axons, when present, are indicated in red. Note that the axons for basket neurons (B) and (U) followed the curvature of the folia in their original sections for a long distance, and thus incorrectly appear, here in the schematic, to extend to or beyond the pial surface. Scale bar: $100 \mu \mathrm{m}$. attenuated dendritic length values. To the extent that we can generalize from the present sample, it appears that, among molecular layer interneurons, the more superficial stellate neurons tended to be smaller than the deeper basket neurons for most dendritic measures, a finding consistent with observations in the cerebral neocortex, where deeper neurons tend to be larger than more superficial neurons (Jacobs et al., 1997, 2001). In the granule cell layer, Lugaro and Golgi neurons were typically similar in overall size (e.g., dendritic Vol and TDL); however, they exhibited vastly different morphologies, as reflected in typically greater MSL values for Lugaro neurons (indicating long, unbranched dendrites) and higher DSC values for Golgi neurons (suggesting a more complex dendritic branching pattern).

Variability in neuronal measures across species was much smaller than that observed for brain mass (64-fold) and cerebellar volume (103-fold). In general, dendritic measurements and soma size tended to be positively correlated with cerebellar volume for most neuron types, although this tendency was skewed by the large size of elephant neurons. There was a 3.08fold difference in soma size among the species in the current sample, with the elephant having the largest values across all neuron types. Moreover, most neuron types were characterized by a 3.5- to 5-fold range of variation in dendritic measures across species. The Lugaro neuron, however, averaged a 7.25-fold variation across species, mainly because of the extraordinary size of Lugaro neurons in the elephant (Maseko et al., 2012a)-note that excluding the elephant data resulted in a 3.19-fold variation in Lugaro neurons. Although the length of Lugaro dendrites in the present sample appears to be within the range of what has been reported in rats (i.e., from 100 to $700 \mu \mathrm{m}$ from the soma; Lainé and Axelrad, 1996), it is the measure of Lugaro dendritic Vol. that especially differentiates the elephant from 


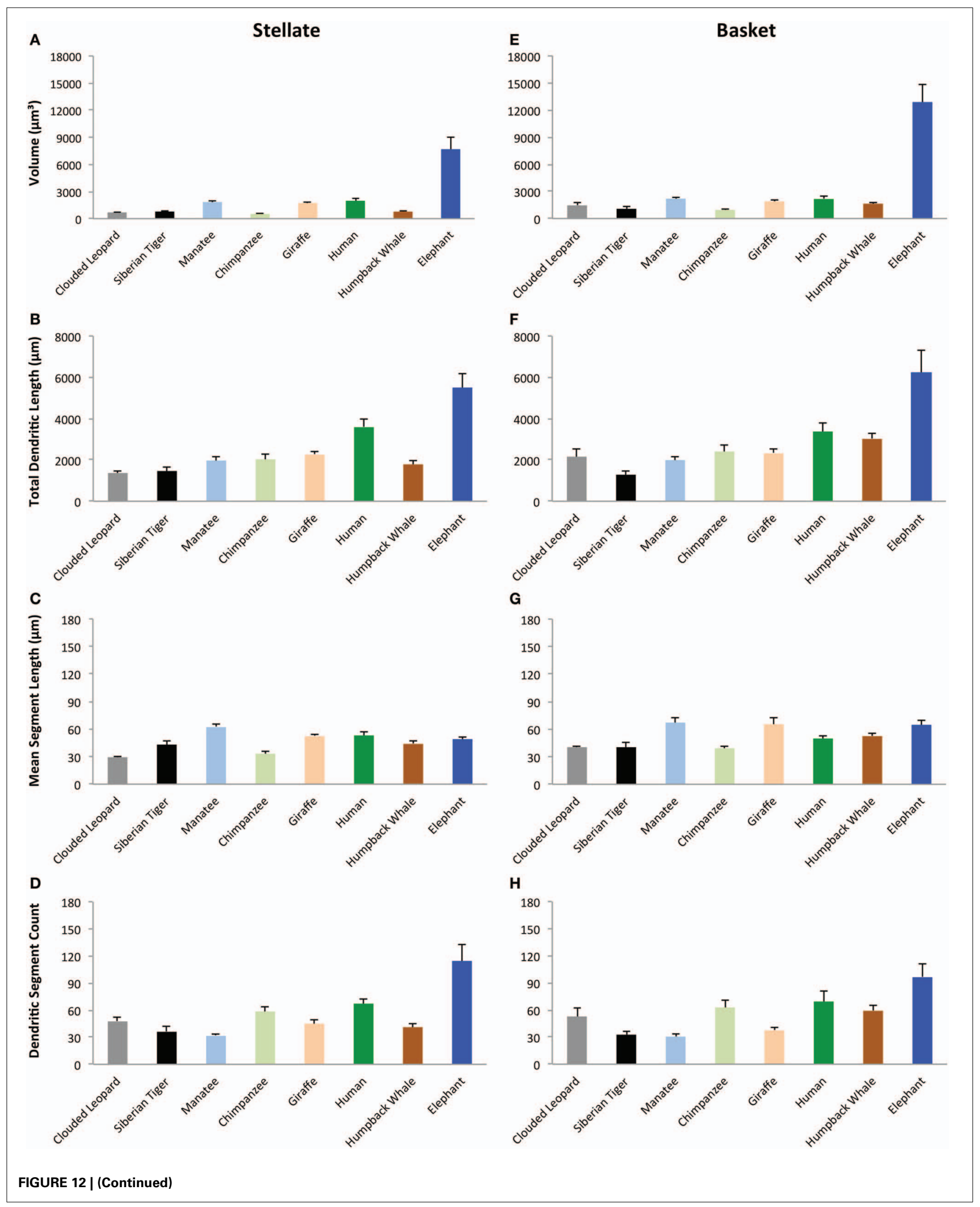




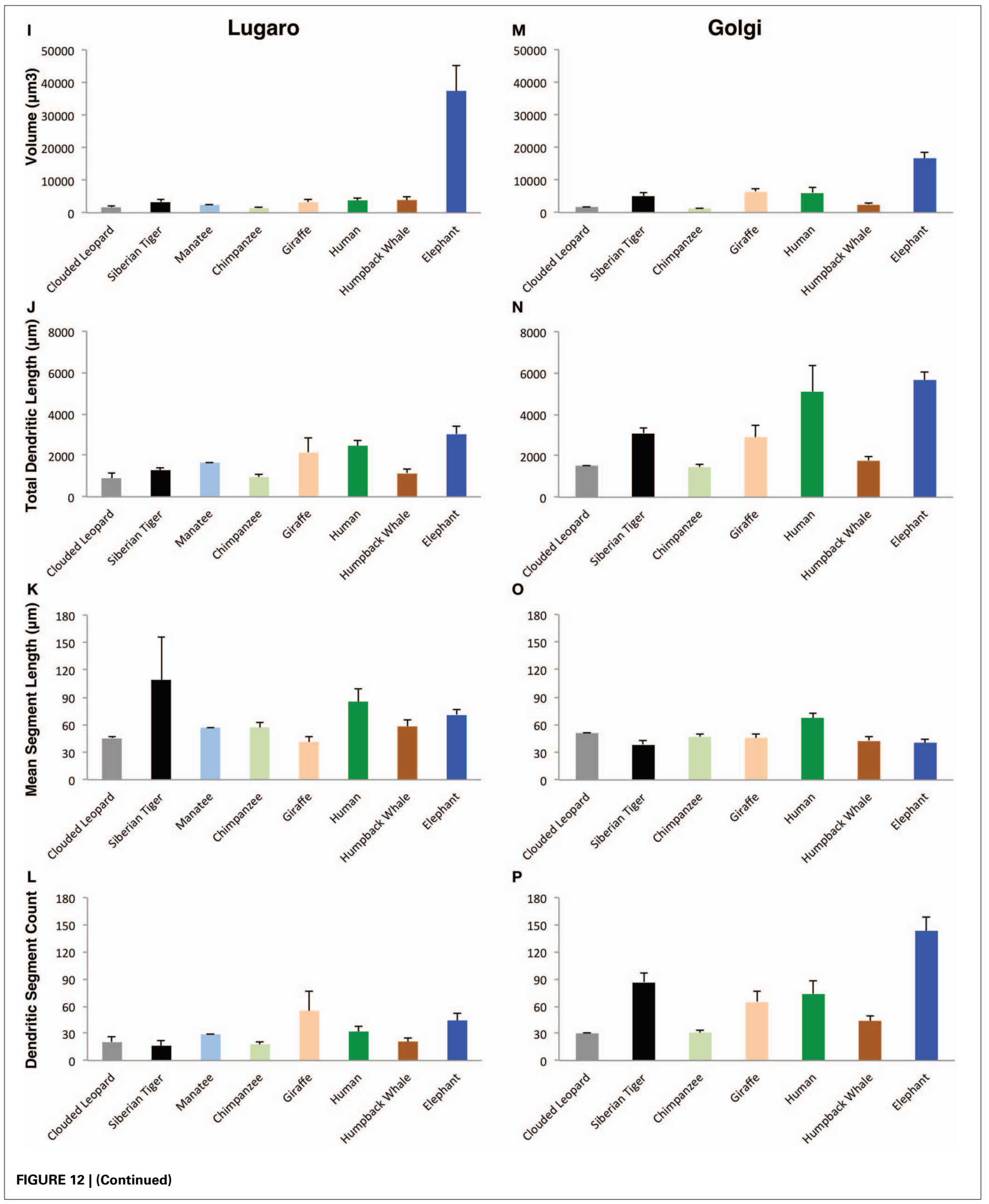




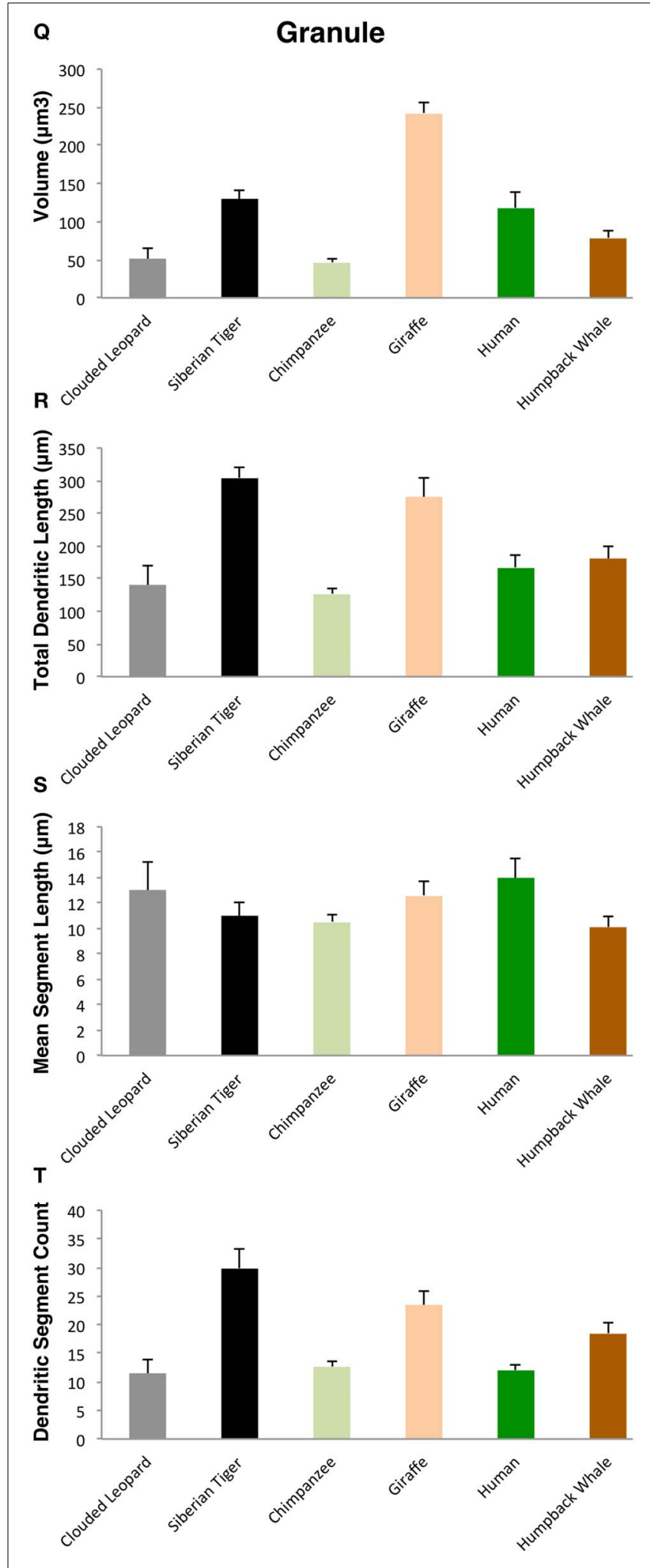

FIGURE 12 | Bar graphs indicated the relative values of four dependent measures (Volume, Total Dendritic Length, Mean Segment Length, and Dendritic Segment Count) for stellate (A-D), basket (E-H), Lugaro (I-L), Golgi (M-P), and granule (Q-T) neurons across the eight species

(Continued)
FIGURE 12 | Continued

in the current study. The eight species are arranged from left to right on the abscissa in a fixed order, from the smallest (clouded leopard) to the largest brain (elephant). Phylogenetic relationships among species are color coded as follows: afrotherians (dark blue = elephant; light blue = manatee); carnivores (black $=$ tiger; gray $=$ leopard); cetartiodactyls (dark brown $=$ humpback; light brown = giraffe); and primates (dark green = human; light green $=$ chimpanzee). Note the following: (1) tortuosity measures are not illustrated here; (2) granule neurons in afrotherians, and Golgi neurons in the manatee are not illustrated here because they did not stain; and (3) the ordinate scale for granule neurons is much smaller than the scale for other neuron types. Error bars $=$ s.e.m.

other species. For dendritic Vol, there was an average 14.43-fold increase across species in the current sample, as opposed to an average 2.81-fold increase for all other dendritic measures. This suggests that dendritic Vol might scale more steeply than other dendritic measures for cross-species comparisons in the cerebellum, an observation that seems to have been confirmed in the current MARSplines analysis, which indicated that dendritic Vol was the most consistently used variable for interspecies differentiation. These findings also appear consistent with the suggestion that there is a positive relationship between brain mass and dendritic extent in the neocortex (Elston et al., 2006; HerculanoHouzel et al., 2006; Sarko et al., 2009; Jacobs et al., 2011; Manger et al., 2013), a corollary being that, similar to the cerebral cortex (Haug, 1967, 1987), neuronal density in the cerebellum appears to be inversely related to brain mass (Lange, 1975; Maseko et al., 2012a).

\section{INFERENTIAL STATISTICAL COMPARISONS ACROSS SPECIES}

One goal of the present investigation was to compare neuronal morphology in the cerebellar cortex across several large brained mammals not previously examined. We tested for species differences using MARSplines analyses, which indicated that there were significant differences in dendritic measures (and soma size) among all species. Moreover, this analysis revealed not only which measures were most important for differentiating individual species, but also the unique combinations and weightings of these measures (Table 4). In future studies, a data set with a much larger number of neurons, and with all neuron types represented for every species, would enable a more detailed evaluation of the relative importance of neuron types (e.g., Lugaro vs. Golgi) to species differentiation. At this point, an interesting question is whether the evolutionary, ecological, and behavioral adaptations that influence brain mass and cerebellar volume, might also shape aspects of the somatodendritic morphology in neurons themselves.

\section{FUNCTIONAL SPECULATIONS}

The large-brained mammals in the current sample represent a diverse range of ecological, somatic, and behavioral adaptations. Here, we can only speculate very generally how these adaptations may relate to factors such as cerebellar volume and neuronal morphology in these species. Insofar as the cerebellum has traditionally been implicated in motor control (Fulton and Dow, 1937; Marr, 1969; Glickstein and Yeo, 1990), the motor system 


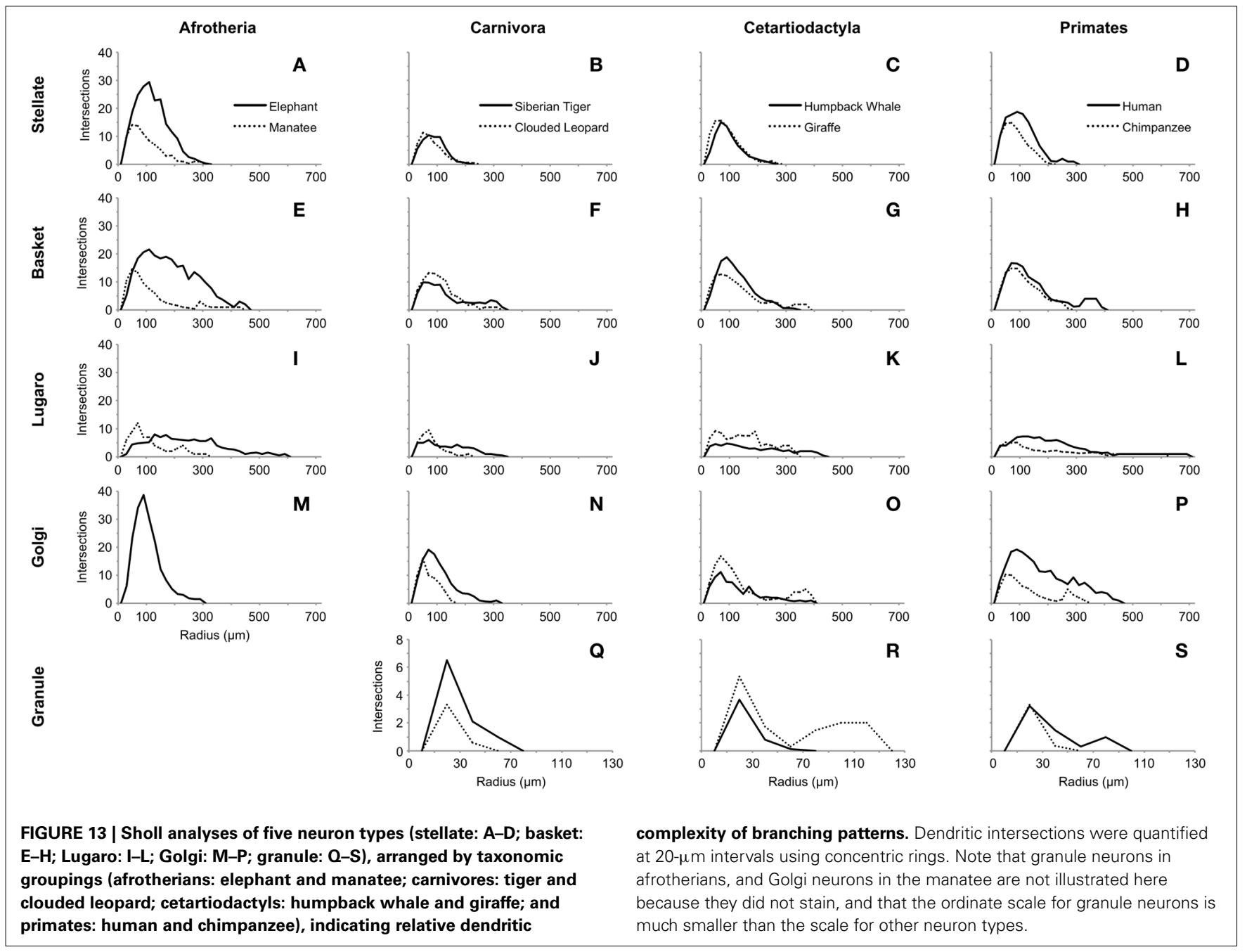

of a particular species is often the initial focal point (Onodera and Hicks, 1999). For example, the elephant possesses the largest absolute and relative cerebellar volume of any mammal investigated to date (Shoshani et al., 2006; Maseko et al., 2012b), a finding typically explained with reference to the fine motor control demands of its trunk (Endo et al., 2001; Maseko et al., 2012b). A more integrative, and perhaps parsimonious, perspective suggests that the cerebellum is not involved exclusively with motor control, but rather that it is engaged in monitoring and adjusting the acquisition of sensory information for the rest of the nervous system (Bower, 1992, 1997; Gao et al., 1996). As such, one factor contributing to the large elephant cerebellum may be the documented importance of the trunk in multi-sensory exploration of the environment (Rasmussen and Munger, 1996; Bagley et al., 2006; Foerder et al., 2011). If this theory is extended to the domain of communication, the auditory-tactile infrasound information perceived through the elephant's feet may also contribute to its enlarged cerebellum (Garstang, 2004; Bouley et al., 2007; Soltis, 2009).

Additional investigations into the sensory role of cerebellum may provide insight into the other species examined in the current study. For example, imaging research has revealed that the lateral cerebellar hemispheres are involved in sensory acquisition and discrimination in humans (Parsons et al., 1997), a finding that may also apply to chimpanzees. Certainly, this is consistent with the expansion of the lateral cerebellum in hominoids relative to other primates (Rilling and Insel, 1998; MacLeod et al., 2003; Rilling, 2006). Electrophysiological research has shown that cats have larger tactile representations of the forelimbs in the lateral cerebellar hemispheres than do rodents (e.g., mice, rats, and guinea pigs) because cats use their forelimbs more for sensory exploration of their environment than do rodents, which depend more on tactile information from the face region (Welker, 1964; Bower, 1997, 2011). Speculatively, cerebellar sensory representations for the manatee may resemble those of rodents insofar as the manatee has a sensitive, perioral tactile system for exploring its aquatic environment (Marshall et al., 1998, 2003; Reep et al., 2001). In contrast, the felines of the current study may be characterized by strong forelimb representation in the lateral cerebellum. Finally, such a sensory focus on cerebellar processing may help clarify why there is a relative increase in cerebellar volume in microchiropterans and odontocete cetaceans (Baron et al., 1996; Marino et al., 2000) vis-à-vis primates (Maseko et al., 2012b). From the perspective of motor control, this observation is difficult 
Table 4 | Correct-incorrect confusion matrices for differentiation of species.

\begin{tabular}{|c|c|c|c|c|c|c|c|c|c|c|c|}
\hline & & \multicolumn{4}{|c|}{ Predicted } & \multicolumn{6}{|c|}{ Predictor importance $^{a}$} \\
\hline & & $99.6 \%$ correct $^{b}$ & Elephant & Other species & Row totalc & Vol & TDL & MSL & DSC & Tor & Soma size \\
\hline \multirow[t]{3}{*}{ African elephant } & \multirow{3}{*}{$\begin{array}{l}D \\
0 \\
D \\
D \\
0 \\
0 \\
0 \\
0\end{array}$} & Elephant & 19 & 1 & 20 & 16 & 17 & 5 & 0 & 8 & 5 \\
\hline & & Other species & 0 & 235 & 235 & & & & & & \\
\hline & & Column total & 19 & 236 & 255 & & & & & & \\
\hline \multirow[t]{4}{*}{ Florida manatee } & \multirow{4}{*}{ 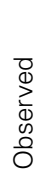 } & $98.5 \%$ correct & Manatee & Other species & Row total & Vol & TDL & MSL & DSC & Tor & Soma size \\
\hline & & Manatee & 11 & 4 & 15 & 5 & 10 & 7 & 0 & 7 & 15 \\
\hline & & Other species & 0 & 254 & 254 & & & & & & \\
\hline & & Column total & 11 & 258 & 269 & & & & & & \\
\hline \multirow[t]{4}{*}{ Siberian tiger } & \multirow{4}{*}{$\begin{array}{l}D \\
D \\
\geq \\
0 \\
\infty \\
0 \\
0 \\
0\end{array}$} & $91.8 \%$ correct & Tiger & Other species & Row total & Vol & TDL & MSL & DSC & Tor & Soma size \\
\hline & & Tiger & 10 & 23 & 33 & 3 & 5 & 16 & 7 & 7 & 3 \\
\hline & & Other species & 3 & 281 & 284 & & & & & & \\
\hline & & Column total & 13 & 304 & 317 & & & & & & \\
\hline \multirow[t]{4}{*}{ Clouded leopard } & \multirow{4}{*}{ 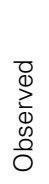 } & $92.1 \%$ correct & Leopard & Other species & Row total & Vol & TDL & MSL & DSC & Tor & Soma size \\
\hline & & Leopard & 8 & 1 & 9 & 9 & 2 & 7 & 0 & 8 & 6 \\
\hline & & Other species & 24 & 284 & 308 & & & & & & \\
\hline & & Column total & 32 & 285 & 317 & & & & & & \\
\hline \multirow[t]{4}{*}{ Humpback whale } & \multirow{4}{*}{$\begin{array}{l}0 \\
0 \\
\mathbb{1} \\
\mathbb{1} \\
\infty \\
\infty \\
0 \\
0\end{array}$} & $89.3 \%$ correct & Whale & Other species & Row total & Vol & TDL & MSL & DSC & Tor & Soma size \\
\hline & & Whale & 13 & 34 & 47 & 2 & 4 & 6 & 3 & 10 & 1 \\
\hline & & Other species & 0 & 270 & 270 & & & & & & \\
\hline & & Column total & 13 & 304 & 317 & & & & & & \\
\hline \multirow[t]{4}{*}{ Giraffe } & \multirow{4}{*}{$\begin{array}{l}0 \\
\mathbb{D} \\
\geq \\
0 \\
\infty \\
0 \\
0 \\
0\end{array}$} & $90.2 \%$ correct & Giraffe & Other species & Row total & Vol & TDL & MSL & DSC & Tor & Soma size \\
\hline & & Giraffe & 27 & 29 & 56 & 7 & 3 & 4 & 6 & 6 & 7 \\
\hline & & Other species & 2 & 259 & 261 & & & & & & \\
\hline & & Column total & 29 & 288 & 317 & & & & & & \\
\hline \multirow[t]{4}{*}{ Human } & \multirow{4}{*}{$\begin{array}{l}\bar{D} \\
\mathbb{1} \\
\mathbb{1} \\
\infty \\
\infty \\
0 \\
0\end{array}$} & $93.7 \%$ correct & Human & Other species & Row total & Vol & TDL & MSL & DSC & Tor & Soma size \\
\hline & & Human & 8 & 20 & 28 & 8 & 9 & 1 & 8 & 6 & 1 \\
\hline & & Other species & 0 & 289 & 289 & & & & & & \\
\hline & & Column total & 8 & 309 & 317 & & & & & & \\
\hline \multirow[t]{4}{*}{ Common chimpanzee } & \multirow{4}{*}{$\begin{array}{l}0 \\
\mathbb{1} \\
\stackrel{1}{1} \\
\mathbb{1} \\
\infty \\
0 \\
0\end{array}$} & $85.5 \%$ correct & Chimpanzee & Other species & Row total & Vol & TDL & MSL & DSC & Tor & Soma size \\
\hline & & Chimpanzee & 63 & 23 & 86 & 6 & 2 & 1 & 3 & 2 & 9 \\
\hline & & Other species & 23 & 208 & 231 & & & & & & \\
\hline & & Column total & 86 & 231 & 317 & & & & & & \\
\hline
\end{tabular}

a Predictor importance indicates how many times each measure appears in the regression analysis for that particular species. See text for more details.

${ }^{b}$ Bold numbers represent the correct predictions. The percentage correct is calculated by dividing the sum of the two bold numbers by the total number of neurons examined. So, for the elephant: $(19+235) / 255=0.996$.

${ }^{c}$ Note that the total number of neurons for elephants and manatees is not 317 , as for all other species, because some neuron types did not stain in the two afrotherians.

to explain insofar as primates arguably have greater fine motor dexterity (Darian-Smith et al., 2007; Kaas, 2008). However, both microchiropterans and odontocetes rely extensively on the coordinated use of sensory surfaces when exploring their environment with echolocation (Norris et al., 1961; Ghose et al., 2006; Surlyke et al., 2009; Akamatsu et al., 2010), and this may contribute to an expansion in cerebellar tissue. By extension, the humpback whale cerebellum may also be affected by the whale's extensive vocal repertoire (Mercado et al., 2010; Garland et al., 2011) which, similar to echolocation, may serve as a type of sonar that provides sensory information about its aquatic world (Frazer and Mercado, 2000). 
Finally, although the basic circuitry of the cerebellar cortex is fairly well documented in a limited number of species, discerning structure-function relationships can be challenging (Sultan and Glickstein, 2007; Schilling et al., 2009). This is especially true when direct electrophysiological experimentation on a species is not possible. There are, however, two morphological findings of particular functional interest in the present study: (1) the distinctive morphology and large size of Lugaro neurons in the elephant cerebellum, and (2) the presence of a palisade dendritic pattern for Purkinje neurons in the humpback whale. With regards to first observation, what remains unclear is whether the elephant Lugaro neurons are functionally connected in the same manner as demonstrated in other species, that is, whether they receive serotonergic input (Dieudonné and Dumoulin, 2000; Geurts et al., 2003), input from Purkinje neuron collaterals (Lainé and Axelrad, 1996; Geurts et al., 2003), and/or whether they project to molecular layer interneurons (Flace et al., 2004; Ambrosi et al., 2007) and to Golgi neurons (Melik-Musyan and Fanardzhyan, 1998; Dumoulin et al., 2001; Crook et al., 2006). The expansive dendritic arbors of elephant Lugaro neuorons would suggest a broad sampling of local input but, because we have no information on their axonal projections, it is unclear to what extent they exert inhibitory feedback on Purkinje neurons, modulate mossy fiber input, and/or contribute to long-term depression (Geurts et al., 2003; Melik-Musyan and Fanardzhyan, 2004). With regards to the humpback whale Purkinje neuron dendrites, any functional speculation would be premature until future research confirms the current, tentative findings. If such Purkinje cell dendritic morphology actually obtains in mysticetes, or in cetaceans in general, then the next question would be whether the acoustic world of cetaceans and the electrosensory system in mormyrids have any neurofunctional commonalities. Only more detailed comparative research will address such issues.

\section{ACKNOWLEDGMENTS}

Partial support for this work was provided by Colorado College's divisional research funds (Bob Jacobs), the James S. McDonnell Foundation (grant 22002078, to Chet C. Sherwood, Patrick R. Hof; grant 220020293 to Chet C. Sherwood), National Science Foundation (BCS-0827531 to Chet C. Sherwood), and the South African National Research Foundation (Paul R. Manger; FA2005033100004). We would also like to thank the Danish Cardiovascular Research Program, especially Emil ToftBrøndum, for allowing us to obtain the specimens of giraffe brains.

\section{REFERENCES}

Adanina, V. (1965). Neurons and connections of the flocculus in dolphins. Arkh. Anat. 56, 29-35.

Akamatsu, T., Wang, D., Wang, K., Li, S., and Dong, S. (2010). Scanning sonar of rolling porpoises during prey capture dives. J. Exp. Biol. 213, 146-152. doi: 10.1242/jeb.037655

Altman, J., and Bayer, S. A. (1977). Time of origin and distribution of a new cell type in the rat cerebellar cortex. Exp. Brain Res. 29, 267-274. doi: 10.1007/BF00237046

Ambrosi, G., Flace, P., Lorusso, L., Girolamo, F., Rizzi, A., Bosco, L., et al. (2007). Non-traditional large neurons in the granular layer of the cerebellar cortex. Eur. J. Histochem. 51, 59-64.
Anderson, K., Bones, B., Robinson, B., Hass, C., Lee, H., Ford, K., et al. (2009). The morphology of supragranular pyramidal neurons in the human insular cortex: a quantitative Golgi study. Cereb. Cortex 19, 2131-2144. doi: 10.1093/cercor/bhn234

Anderson, K., Yamamoto, E., Kaplan, J., Hannan, M., and Jacobs, B. (2010). Neurolucida Lucivid vs. Neurolucida camera: a quantitative and qualitative comparison of three-dimensional neuronal reconstructions. J. Neurosci. Methods 186, 209-214. doi: 10.1016/j.jneumeth.2009.11.024

Bagley, K. R., Goodwin, T. E., Rasmusen, L. E. L., and Schulte, B. A. (2006). Male African elephants, Loxodonta africana, can distinguish oestous status via urinary signals. Animal Behav. 71, 1439-1445. doi: 10.1016/j.anbehav.2006.01.003

Baron, G., Stephan, H., and Frahm, H. D. (1996). Comparative Neurobiology in Chiroptera. Basel: Birkhauser Verlag.

Bell, B. A., Ferron, J. M., and Kromrey, J. D. (2008). "Cluster size in multilevel models: the impact of sparse data structures on point and interval estimates in two-level models," in JSM Proceedings, Section on Survey Research Methods, 1122-1129. Available Online at: http://www.amstat.org/Sections/ Srms/Proceedings/y2008/Files/300933.pdf

Berk, R. A., and Freedman, D. A. (2003). "Statistical assumptions as empirical commitments," in Punishment and Social Control: Essays in Honor of Sheldon L. Messinger, eds S. Cohen and T. G. Blomberg (New Brunswick, NJ: Aldine de Gruyter), 235-254.

Bishop, G. A. (1993). An analysis of HRP-filled basket cell axons in the cat's cerebellum. I. Morphometry and configuration. Anat. Embrol. 188, 287-297.

Bok, S. T. (1959). Histonomy of the Cerebral Cortex. Amsterdam: Elsevier.

Bolk, L. (1906). Das Cerebellum der Säugetiere: eine Vergleichende Anatomische Untersuchung. Haarlem: Fischer.

Bouley, D. M., Alarcon, C. N., Hildebrandt, T., and O'Connell-Rodwell, C. E. (2007). The distribution, density and three-dimensional histomorphology of Pacinian corpuscles in the foot of the Asian elephant (Elephas maximus) and their potential role in seismic communication. J. Anat. 211, 428-435. doi: 10.1111/j.1469-7580.2007.00792.x

Bower, J. M. (1992). Is the cerebellum a motor control device? Behav. Brain Sci. 15, 714-715.

Bower, J. M. (1997). Is the cerebellum sensory for motor's sake, or motor for sensory's sake: the view from the whiskers of a rat? Prog. Brain Res. 114, 483-516. doi: 10.1016/S0079-6123(08)63381-6

Bower, J. M. (2011). Functional implications of tactile projection patterns to the lateral hemispheres of the cerebellum of the albino rat: the legacy of Wally Welker. Ann. N.Y. Acad. Sci. 1225, 130-141. doi: 10.1111/j.1749-6632.2011.06020.x

Braak, E., and Braak, H. (1983). On three types of large nerve cells in the granular layer of the human cerebellar cortex. Anat. Embryol. 166, 67-86. doi: 10.1007/BF00317945

Braak, H., and Braak, E. (1985). Golgi preparations as a tool in neuropathology with particular reference to investigations of the human telencephalic cortex. Prog. Neurobiol. 25, 93-139. doi: 10.1016/0301-0082(85)90001-2

Breathnach, A. S. (1955). The surface features of the brain of the humpback whale (Megaptera novaeangliae). J. Anat. 89, 343-352.

Buell, S. J. (1982). Golgi-Cox and rapid Golgi methods as applied to autopsied human brain tissue: widely disparate results. J. Neuropathol. Exp. Neurol. 41, 500-507. doi: 10.1097/00005072-198209000-00003

Calvet, M.-C., and Calvet, J. (1984). Computer assisted analysis of HRP labeled and Golgi stained Purkinje neurons. Prog. Neurobiol. 23, 251-272. doi: 10.1016/0301-0082(84)90006-6

Chan-Palay, V., and Palay S. L. (1970). Interrelations of basket cell axons and climbing fibers in the cerebellar cortex of the rat. Z. Anat. Entwickl-Gesch. 131, 191-227. doi: 10.1007/BF00523377

Chan-Palay, V., and Palay S. L. (1972). The stellate cells of the rat's cerebellar cortex. Z. Anat. Entwickl-Gesch. 136, 224-248. doi: 10.1007/BF00519180

Christ, H. (1985). Fusiform nerve cells of the granular layer in the cerebellar cortex of the baboon. Neurosci. Lett. 56, 195-198. doi: 10.1016/0304-3940(85)90128-4

Clark, D. A., Mitra, P. P., and Wang, S. S.-H. (2001). Scalable architecture in mammalian brains. Nature 411, 189-193. doi: 10.1038/35075564

Crook, J., Hendrickson, A., and Robinson, F. R. (2006). Co-localization of glycine and GABA immuoreactivity in interneurons in macaca monkey cerebellar cortex. Neuroscience 141, 1951-1959. doi: 10.1016/j.neuroscience.2006.05.012

Darian-Smith, I., Galea, M. P., and Darian-Smith, C. (2007). Manual dexterity: how does the cerebral cortex contribute? Clin. Exp. Pharmacol. Physiol. 23, 948-956. doi: 10.1111/j.1440-1681.1996.tb01147.x 
Dean, P., Porrill, J., Ekerot, C.-F., and Jörntell, H. (2010). The cerebellar microcircuit as an adaptive filter: experimental and computational evidence. Nat. Rev. Neurosci. 11, 30-43. doi: 10.1038/nrn2756

Dell, L.-H., Patzke, N., Bhagwandin, A., Bux, F., Fuxe, K., Barber, G., et al. (2012). Organization and number of orexinergic neurons in the hypothalamus of two species of Cetartiodactyla: a comparison of giraffe (Giraffa camelopardalis) and harbour porpoise (Phocoena phocoena). J. Chem. Neuroanat. 44, 98-109. doi: 10.1016/j.jchemneu.2012.06.001

de Ruiter, J. P. (1983). The influence of post-mortem fixation delay on the reliability of the Golgi silver impregnation. Brain Res. 266, 143-147. doi: 10.1016/00068993(83)91317-3

Dieudonné, S., and Dumoulin, A. (2000). Serotonin-driven long-range inhibitory connections in the cerebellar cortex. J. Neurosci. 20, 1837-1848.

Dumoulin, A., Triller, A., and Dieudonné, S. (2001). IPSC kinetics at identified GABAergic and mixed GABAergic and glycinergic synapses onto cerebellar Golgi cells. J. Neurosci. 21, 6045-6047.

Elston, G. N., Benavides-Piccione, R., Elston, A., Zietsch, B., Defelipe, J., Manger, P., et al. (2006). Specializations of the granular prefrontal cortex of primates: implications for cognitive processing. Anat. Rec. 288, 26-35. doi: 10.1002/ar. a. 20278

Endo, H., Hayashi, Y., Komiya, T., Narushima, E., and Sasako, M. (2001). Muscle architecture of the elongated nose in the Asian elephant (Elephas maximus). J. Vet. Med. Sci. 63, 533-537. doi: 10.1292/jvms.63.533

Flace, P., Benagiano, V., Lorusso, L., Girolamo, F., Rizzi, A., Virgintino, D., et al. (2004). Glutamic acid decarboxylase immunoreative large neurons types in the granular layer of the human cerebellar cortex. Anat. Embrol. 208, 55-64. doi: 10.1007/s00429-003-0374-X

Foerder, P., Galloway, M., Barthel, T., Moore, D. E. III., and Reiss, D. (2011). Insightful problem solving in an Asian elephant. PLOS ONE 6:e23251. doi: 10.1371/journal.pone.0023251

Foster, R. E., and Peterson, B. E. (1986). The inferior olivary complex of guinea pig: cytoarchitecture and cellular morphology. Brain Res. Bull. 17, 785-800. doi: 10.1016/0361-9230(86)90090-0

Fox, C. A., Hillman, D. E., Siegesmund, K. A., and Dutta, C. R. (1967). The primate cerebellar cortex: a Golgi and electron microscopic study. Prog. Brain Res. 25, 174-225. doi: 10.1016/S0079-6123(08)60965-6

Frazer, L. N., and Mercado, E. 3rd. (2000). A sonar model for humpback whale song. IEEE J. Ocean. Eng. 25, 160-182. doi: 10.1109/48.820748

Freedman, D. A. (2004). "Sampling" in the Encyclopedia of Social Science Research Methods, Vol. 3, eds M. Lewis-Beck, A. Bryman, and T. F. Liao (Thousand Oaks, CA: Sage Publications), 986-990.

Friedland, D. R., Los, J. G., and Ryugo, D. K. (2006). A modified Golgi staining protocol for use in the human brain stem and cerebellum. J. Neurosci. Methods 150, 90-95. doi: 10.1016/j.jneumeth.2005.06.004

Friedman, J. H. (1991). Multivariate adaptive regression splines. Ann Stat 19, 1-67. doi: 10.1214/aos/1176347963

Fulton, J. F., and Dow, R. S. (1937). The cerebellum: a summary of functional localization. Yale J. Biol. Med. 10, 89-119.

Gao, J. H., Parsons, L. M., Bower, J. M., Xiong, J., Li, J., and Fox, P. T. (1996). Cerebellum implicated in sensory acquisition and discrimination rather than motor control. Science 272, 545-547. doi: 10.1126/science.272. 5261.545

Garland, E. C., Goldizen, A. W., Rekdahl, M. L., Constantine, R., Garrigue, C., Hauser, N. D., et al. (2011). Dynamic horizontal cultural transmission of humpback whale song at the ocean basin scale. Curr. Biol. 21, 687-691. doi: 10.1016/j.cub.2011.03.019

Garstang, M. (2004). Long-distance, low-frequency elephant communication. J. Comp. Physiol. A. Neuroethol. Sens. Neural Behav. Physiol. 190, 791-805. doi: 10.1007/s00359-004-0553-0

Geurts, F. J., Schutter, E. D., and Dieudonné, S. (2003). Unravelling the cerebellar cortex: cytology and cellular physiology of large-sized interneurons in the granular layer. Cerebellum 2, 290-299. doi: 10.1080/14734220310011948

Ghose, K., Horiuchi, T. K., Krishnaprasad, P. S., and Moss, C. F. (2006). Echolocating bats use a nearly time-optimal strategy to intercept prey. PLoS Biol. 4:e108. doi: 10.1371/journal.pbio.0040108

Glickstein, M., and Yeo, C. (1990). The cerebellum and motor learning. J. Cog. Neurosci. 2, 69-80. doi: 10.1162/jocn.1990.2.2.69

Golgi, C. (1874). Sulla fina anatomia del cevelletto umano. Arch. Ital. per le Mal. Nerv. 2, 90-107.
Halavi, M., Hamilton, K. A., Parekh, R., and Ascoli, G. A. (2012). Digital reconstructions on neuronal morphology: three decades of research trends. Front. Neurosci. 6:49. doi: 10.3389/fnins.2012.00049

Han, V. Z., Meek, J., Campbell, H. R., and Bell, C. C. (2006). Cell morphology and circuitry in the central lobes of the mormyrid cerebellum. J. Comp. Neurol. 497, 309-325. doi: 10.1002/cne.20983

Hastie, T., Tibshirani, R., and Friedman, J. (2009). The Elements of Statistical Learning: Data Mining, Inference, and Prediction, 2nd Edn. New York, NY: Springer-Verlag. doi: 10.1007/978-0-387-84858-7

Haug, H. (1967). Zytoarchitektonische Untersuchungen an der Hinrnrinde des Elefanten. Anat. Anz. 120, 331-337.

Haug, H. (1987). Brain sizes, surfaces, and neuronal sizes of the cortex cerebri: a stereological investigation of man and his variability and a comparison with some mammals (primates, whales, marsupials, insectivores, and one elephant). Am. J. Anat. 180, 126-142. doi: 10.1002/aja.1001800203

Herculano-Houzel, S., Mota, B., and Lent, R. (2006). Cellular scaling rules for rodent brains. Proc. Natl. Acad. Sci. U.S.A. 103, 12138-12143. doi: 10.1073/pnas. 0604911103

Iwaniuk, A. N., Hurd, P. L., and Wylie, D. R. W. (2006). The comparative morphology of the cerebellum in Caprimulgiform birds: evolutionary and functional implications. Brain Behav. Evol. 67, 53-68. doi: 10.1159/000089120

Jacobs, B., Lubs, J., Hannan, M., Anderson, K., Butti, C., Sherwood, C. C., et al. (2011). Neuronal morphology in the African elephant (Loxodonta africana) neocortex. Brain Struct. Funct. 215, 273-298. doi: 10.1007/s00429-010-0288-3

Jacobs, B., Schall, M., and Scheibel, A. B. (1993). A quantitative dendritic analysis of Wernicke's area in humans. II. Gender, hemispheric, and environmental factors. J. Comp. Neurol. 327, 97-111. doi: 10.1002/cne.903270108

Jacobs, B., Driscoll, L., and Schall, M. (1997). Life-span dendritic and spine changes in areas 10 and 18 of human cortex: a quantitative Golgi study. J. Comp. Neurol. 386, 661-680. doi: 10.1002/(SICI)1096-9861(19971006)386:4< 661::AID-CNE11>3.0.CO;2-N

Jacobs, B., Schall M., Prather, M., Kapler, E., Driscoll, L., and Baca, S. (2001). Regional dendritic and spine variation in human cerebral cortex: a quantitative Golgi study. Cereb. Cortex 11, 558-571. doi: 10.1093/cercor/11.6.558

Jacobs, B., and Scheibel, A. B. (1993). A quantitative dendritic analysis of Wernicke's area in humans. I. Lifespan changes. J. Comp. Neurol. 327, 83-96. doi: $10.1002 / \mathrm{cne} .903270107$

Jacobs, B, and Scheibel, A. B. (2002). "Regional dendritic variation in primate cortical pyramidal cells," in Cortical Areas: Unity and Diversity (Conceptual Advances in Brain Research Series), eds A. Schüz and R. Miller (London: Taylor and Francis), 111-131.

Kaas, J. H. (2008). The evolution of the complex sensory and motor systems of the human brain. Brain Res. Bull. 75, 384-390. doi: 10.1016/j.brainresbull.2007.10.009

Lainé, J., and Axelrad, H. (1994). The candelabrum cell: a new interneuron in the cerebellar cortex. J. Comp. Neurol. 339, 159-173. doi: 10.1002/cne.903390202

Lainé, J., and Axelrad, H. (1996). Morphology of the Golgi-Impregnated Lugaro cell in the rat cerebellar cortex: a reappraisal with a description of its axon. J. Comp. Neurol. 375, 618-640.

Lainé, J., and Axelrad, H. (2002). Extending the cerebellar Lugaro cell class. Neuroscience 115, 363-374. doi: 10.1016/S0306-4522(02)00421-9

Landau, E. (1933). La cellule synarmotique dans le cervelet humain. Arch. Anat. 17, 273-285.

Lange, W. (1975). Cell number and cell density in the cerebellar cortex of man and some other mammals. Cell Tissue Res. 157, 115-124. doi: 10.1007/BF002 23234

Larsell, O. (1970). The Comparative Anatomy and Histology of the Cerebellum from Monotremes through Apes. Minneapolis, MN: The University of Minnesota.

Larsell, O., and Jansen, J. (1972). The Comparative Anatomy and Histology of the Cerebellum: the Human Cerebellum, Cerebellar Connections, and Cerebellar Cortex. Minneapolis, MN: The University of Minnesota.

Leto, K., Carletti, B., Williams, I. M., Magrassi, L., and Rossi, F. (2006). Different types of cerebellar GABAergic interneurons originate from a common pool of multipotent progenitor cells. J. Neurosci. 26, 11682-11694. doi: 10.1523/JNEUROSCI.3656-06.2006

Lu, D., He, L., Xiang, W., Ai, W.-M., Cao, Y., Wang, X.-S., et al. (2013). Somal and dendritic development of human CA3 pyramidal neurons from midgestation to middle childhood: a quantitative Golgi study. Anat. Rec. 296, 123-132. doi: 10.1002/ar.22616 
Lugaro, E. (1894). Sulle connessioni tra gli elementi nervosi della corteccia cerebellare con considerazioni generali sul significato fisiologico dei rapporti tra gli elementi nervosi. Riv. Sper. Fren. Med. Leg. 20, 297-331.

MacLeod, C. E., Zilles, K., Schleicher, A., Rilling, J. K., and Gibson, K. R. (2003). Expansion of the neocerebellum in Hominoidea. J. Hum. Evol. 44, 401-429. doi: 10.1016/S0047-2484(03)00028-9

Manger, P. R., Pillay, P., Madeko, B. C., Bhagwandin, A., Gravett, N., Moon, D.-J., et al. (2009). Acquisition of brains from the African elephant (Loxodonta africana): perfusion-fixation and dissection. J. Neurosci. Methods 179, 16-21. doi: 10.1016/jneumeth.2009.01.001

Manger, P. R., Cort, J., Ebrahim, N., Goodman, A., Henning, J., Karolia, M., et al. (2008). Is 21st century neuroscience too focused on the rat/mouse model of the brain function and dysfunction? Front. Neuroanat. 2:5. doi: 10.3389/neuro.05.005.2008

Manger, P. R., Spocter, M. A., and Patzke, N. (2013). The evolutions of large brain size in mammals: the "over-700-gram club quartet." Brain Behav. Evol. 82, 68-78. doi: 10.1159/000352056

Marr, D. (1969). A theory of cerebellar cortex. J. Physiol. 202, 437-470.

Marino, L., Rilling, J. K., Lin, S. K., and Ridgway, S. H. (2000). Relative volume of the cerebellum in dolphins and comparison with anthropoid primates. Brain Behav. Evol. 56, 204-211. doi: 10.1159/000047205

Marshall, C. D., Clark, L. A., and Reep, R. L. (1998). The muscular hydrostat of the Florida manatee (Trichechus manatus latirostris): a functional morphological model of perioral bristle use. Mar. Mamm. Sci. 14, 290-303. doi: 10.111/j.1748-7692.1998.tb00717.x

Marshall, C. D., Maeda, H., Iwata, M., Furuta, M., Asano, S., Rosas, F., et al (2003). Orofacial morphology and feeding behaviour of the dugong, Amazonian, West African and Antillean manatees (Mammalia: Sirenia): functional morphology of the muscular-vibrissal complex. J. Zool. Lond. 259, 245-260. doi: 10.1017/ S09528369022003205

Maseko, B. C., Jacobs, B., Spocter, M. A., Sherwood, C. C., Hof, P. R., and Manger, P. R. (2012a). Qualitative and quantitative aspects of the microanatomy of the African elephant cerebellar cortex. Brain Behav. Evol. 81, 40-55. doi: $10.1159 / 000345565$

Maseko, B. C., Spocter, M. A., Haagensen, M. M., and Manger, P. R. (2012b). Elephants have relatively the largest cerebellum size of mammals. Anat. Rec. 295, 661-672. doi: 10.1002/ar.22425

Mavroudis, I. A., Manani, M. G., Petrides, F., Petsoglou, K., Njau, S. D., Costa, V. G., et al. (2013). Dendritic and spinal pathology of the Purkinje cells from the human cerebellar vermis in Alzheimer's disease. Psychiat. Danub. 25, 221-226.

Meek, J. (1992). Comparative aspects of cerebellar organization: from mormyrids to mammals. Eur. J. Morphol. 30, 37-51.

Meek, J., and Nieuwenhuys, R. (1991). Palisade pattern of mormyrid Purkinje cells: a correlated light and electron microscopic study. J. Comp. Neurol. 306, 156-192. doi: 10.1002/cne.903060111

Meek, J., Yang, J. Y., Han, V. Z., and Bell, C. C. (2008). Morphological analysis of the mormyrid cerebellum using immunohistochemistry, with emphasis on the unusual neuronal organization of the valvula. J. Comp. Neurol. 510, 396-421. doi: 10.1002/cne.21809

Melik-Musyan, A. B., and Fanardzhyan, V. V. (1998). Histological identification of Lugaro cells in the cat cerebellum. Neurosci. Behav. Physiol. 28, 486-489. doi: 10.1007/BF02463006

Melik-Musyan, A. B., and Fanardzhyan, V. V. (2004). Morphological characteristics of Lugaro cells in the cerebellar cortex. Neurosci. Behav. Physiol. 34, 633-638. doi: 10.1023/B:NEAB.0000028297.30474.f9

Mercado, E. 3rd., Schneider, J. N., Pack, A. A., and Herman, L. M. (2010). Sound production by singing humpback whales. J. Acoust. Soc. Am. 178, 2678-2691. doi: $10.1121 / 1.3309453$

Milatovic, D., Montine, T. J., Zaja-Milatovic, S., Madison, J. L., Bowman, A. B., and Aschner, M. (2010). Morphometric analysis in neurogenerative disorders. Curr. Protoc. Toxicol. 12, 1-14. doi:10.1002/0471140856.tx1216s43

Murakami, T., and Morita, Y. (1987). Morphology and distribution of the projection neurons in the cerebellum in a Teleost, Sebastiscus marmoratus. J. Comp. Neurol. 256, 607-623. doi: 10.1002/cne.902560413

Nicholson, C., and Llinas, R. (1971). Field potentials in the alligator cerebellum and theory of their relationship to Purkinje cell dendritic spikes. J. Neurophysiol. 34, 509-531.

Nieuwenhuys, R., and Nicholson, C. (1967). The cerebellum of mormyrids. Nature 215, 764-765. doi: $10.1038 / 215764 \mathrm{a} 0$
Norris, K. S., Prescott, J. H., Asa-Dorian, P. V., and Perkins, P. (1961). An experimental demonstration of echo-location behavior in the porpoise, Tursiops truncatus (Montagu). Biol. Bull. 120, 163-176. doi: 10.2307/ 1539374

Ohm, T. G., and Diekmann, S. (1994). The use of Lucifer Yellow and Mini-Ruby for intracellular staining in fixed brain tissue: methodological considerations evaluated in rat and human autopsy brains. J. Neurosci. Methods 55, 105-110. doi: 10.1016/0165-0270(94)90046-9

O’Leary, J. L., Petty, J., Smith, J. M., O’Leary, M., and Inukai, J. (1968). Cerebellar cortex of rat and other animals: a structural and ultrastructural study. J. Comp. Neurol. 134, 401-432.

Onodera, S., and Hicks, P. T. (1999). Review: evolution of the motor system: why the elephant's trunk works like a human's hand. Neuroscientist 5, 217-226. doi: 10.1177/107385849900500411

Palay, S. L., and Chan-Palay, V. (1974). Cerebellar Cortex: Cytology and Organization. Berlin: Springer-Verlag. doi: 10.1007/978-3-642-65581-4

Parsons, L. M., Bower, J. M., Gao, J.-H., Xiong, J., Li, J., and Fox, P. T. (1997). Lateral cerebellar hemispheres actively support sensory acquisition and discrimination rather than motor control. Learn. Mem. 4, 49-62. doi: 10.1101/lm. 4.1 .49

Rakic, P. (1972). Extrinsic cytological determinants of basket and stellate cell dendritic pattern in the cerebellar molecular layer. J. Comp. Neurol. 146, 335-354. doi: 10.1002/cne.901460304

Ramón y Cajal, S. (1909, 1911). Histologie du Système Nerveux de l'Homme et des Vertébrés. Azoulay L. (transl.) Paris: Maloine.

Rapp, M., Segev, I., and Yarom, Y. (1994). Physiology, morphology and detailed passive models of guinea-pig cerebellar Purkinje cells. J. Physiol. 474, 101-118.

Rasmussen, L. E. L., and Munger, B. L. (1996). The sensorineural specializations of the trunk tip (finger) of the Asian elephant, Elephas maximus. Anat. Rec. 246, 127-134. doi: 10.1002/(SICI)1097-0185(199609)246:1\&lt;127::AID-AR14\&gt; 3.0.CO;2-R

Reep, R. L., and O'Shea, T. J. (1990). Regional brain morphometry and lissencephaly in the Sirenia. Brain Behav. Evol. 35, 185-194. doi: $10.1159 / 000115866$

Reep, R. L., Stoll, M. L., Marshall, C. D., Homer, B. L., and Samuelson, D. A. (2001). Microanatomy of facial vibrissae in the Florida manatee: the basis for specialized sensory function and oripulation. Brain Behav. Evol. 58, 1-14. doi: $10.1159 / 000047257$

Rilling, J. K. (2006). Human and nonhman primate brains: are they allometrically scaled versions of the same design? Evol. Anthropol. 15, 65-77. doi: 10.1002/evan.20095

Rilling, J. K., and Insel, T. R. (1998). Evolution of the cerebellum in primates: differences in relative volume among monkeys, apes and humans. Brain Behav. Evol. 52, 308-314. doi: 10.1159/000006575

Roitman, M. F., Na, E., Anderson, G., Jones, T. A., and Bernstein, I. L. (2002). Induction of a salt appetite alters dendritic morphology in nucleus accumbens and sensitizes rats to amphetamine. J. Neurosci. 22, RC225 (1-5).

Roth, A., and Häuser, M. (2001). Compartmental models of rat cerebellar Purkinje cells based on simultaneous somatic and dendritic patch-clamp recordings. J. Physiol. 535, 445-472. doi: 10.1111/j.1469-7793.2001.00445.x

Sahin, M., and Hockfield, S. (1990). Molecular identification of the Lugaro cell in the cat cerebellar cortex. J. Comp Neurol. 301, 575-584.

Sarko, D. K., Catania, K. C., Leitch, D. B., Kaas, J. H., and Herculano-Houzel, S. (2009). Cellular scaling rules of insectivore brains. Front. Neuroanat. 3:8. doi: 10.3389/neuro.05.008.2009

Sawada, Y., Kajiwara, G., Iizuka, A., Takayama, K., Shuvaev, A. N., Koyama, C., et al. (2010). High transgene expression by lentiviral vectors causes maldevelopment of Purkinje cells in vivo. Cerebellum 9, 291-302. doi: 10.1007/s12311-0100161-1

Schadé, J. P., and Caveness, W. F. (1968). IV. Alteration in dendritic organization. Brain Res. 7, 59-86.

Scheibel, M. E., and Scheibel, A. B. (1978). "The methods of Golgi," in Neuroanatomical Research Techniques, ed R. T. Robertson (New York, NY: Academic Press), 89-114. doi: 10.1016/B978-0-12-590350-9. 50011-2

Schilling, K., Oberdick, J., Rossi, R., and Baader, S. L. (2009). Besides Purkinje cells and granule neurons: an appraisal of the cell biology of the interneurons of the cerebellar cortex. Histochem. Cell Biol. 130, 601-615. doi: 10.1007/s00418-0080483-y 
Simat, M., Parpan, F., and Fritschy, J.-M. (2007). Heterogeneity of glycinergic and gabaergic interneurons in the granule cell layer of mouse cerebellum. J. Comp. Neurol. 500, 71-83. doi: 10.1002/cne.21142

Sholl, D. A. (1953). Dendritic organization of the neurons of the visual and motor cortices of the cat. J. Anat. 87, 387-406.

Shoshani, J., Kupsky, W. J., and Marchant, G. H. (2006). Elephant brain part I: gross morphology, functions, comparative anatomy, and evolution. Brain Res. Bull. 70, 124-157. doi: 10.1016/j.brainresbull.2006.03.016

Smaers, J. B., Steele, J., and Zilles, K. (2011). Modeling the evolution of corticocerebellar systems in primates. Ann. N.Y. Acad. Sci. 1225, 176-190. doi: 10.1111/j.1749-6632.2011.06003.x

Soltis, J. (2009). Vocal communication in African elephants (Loxodonta africana). Zoo Biol. 28, 1-18. doi: 10.1002/zoo.20251

Sultan, F., and Bower, J. M. (1998). Quantitative Golgi study of the rat cerebellar molecular layer interneurons using principal component analysis. J. Comp. Neurol. 393, 353-373. doi: 10.1002/(SICI)1096-9861(19980413)393:33.3. $\mathrm{CO} ; 2-7$

Sultan, F., and Braitenberg, V. (1993). Shapes and sizes of different mammalian cerebella. A study in quantitative comparative neuroanatomy. J. Hirnforsch. 1, 79-92.

Sultan, F., and Glickstein, M. (2007). The cerebellum: comparative and animal studies. Cerebellum 6, 168-176. doi: 10.1080/14734220701332486

Surlyke, A., Ghose, K., and Moss, C. F. (2009). Acoustic scanning of natural scenes by echolocation in the big brown bat, Eptesicus fuscus. J. Exp. Biol. 212, 1011-1020. doi: 10.1242/jeb.024620

Ulfhake, B. (1984). A morphometric study of the soma, first-order dendrites and proximal axon of cat lumbar $\alpha$-motoneurones intracellularly labeled with HRP. Exp. Brain Res. 56, 327-334. doi: 10.1007/BF00236288

Uylings, H. B. M., Ruiz-Marcos, A., and van Pelt, J. (1986). The metric analysis of three-dimensional dendritic tree patterns: a methodological review. J. Neurosci. Methods 18, 127-151. doi: 10.1016/0165-0270(86)90116-0

Welker, W. (1964). Analysis of the sniffing behavior of the albino rat. Behavior 22, 223-244. doi: 10.1163/156853964X00030
Wen, Q., Stepanyants, A., Elston, G. N., Grosberg, A. Y., and Chklovskii, D. B. (2009). Maximization of the connectivity repertoire as a statistical principle governing the shapes of dendritic arbors. Proc. Natl. Acad. Sci. U.S.A. 106 12536-12541. doi: 10.1073/pnas.0901530106

Williams, M. N., Grajales, C. A. G., and Kurkiewicz, D. (2013). Assumptions of multiple regression: correcting two misconceptions. Pract. Assess. Res. Eval. 18. Available online at: http://pareonline.net/getvn.asp? $\mathrm{v}=18 \& \mathrm{n}=11$

Williams, R. S. Ferrante, R. J., and Caviness, V. S. Jr. (1978). The Golgi rapid method in clinical neuropathology: morphological consequences of suboptimal fixation. J. Neuropath. Exp. Neurol. 37, 13-33. doi: 10.1097/00005072-19780 1000-00002

Wu, K.-Y., Zhou, X.-P., and Luo, Z.-G. (2010). Geranylgeranyltransferase I is essential for dendritic development of cerebellar Purkinje cells. Mol. Brain 3:18. doi: 10.1186/1756-6606-3-18

Conflict of Interest Statement: The authors declare that the research was conducted in the absence of any commercial or financial relationships that could be construed as a potential conflict of interest.

Received: 31 October 2013; accepted: 01 April 2014; published online: 23 April 2014. Citation: Jacobs B, Johnson NL, Wahl D, Schall M, Maseko BC, Lewandowski A, Raghanti MA, Wicinski B, Butti C, Hopkins WD, Bertelsen MF, Walsh T, Roberts $J R$, Reep RL, Hof PR, Sherwood CC and Manger PR (2014) Comparative neuronal morphology of the cerebellar cortex in afrotherians, carnivores, cetartiodactyls, and primates. Front. Neuroanat. 8:24. doi: 10.3389/fnana.2014.00024

This article was submitted to the journal Frontiers in Neuroanatomy.

Copyright (C) 2014 Jacobs, Johnson, Wahl, Schall, Maseko, Lewandowski, Raghanti, Wicinski, Butti, Hopkins, Bertelsen, Walsh, Roberts, Reep, Hof, Sherwood and Manger. This is an open-access article distributed under the terms of the Creative Commons Attribution License (CC BY). The use, distribution or reproduction in other forums is permitted, provided the original author(s) or licensor are credited and that the original publication in this journal is cited, in accordance with accepted academic practice. No use, distribution or reproduction is permitted which does not comply with these terms. 